\title{
Mechanical switching of ferroelectric rubber
}

\author{
J. M. Adams ${ }^{1}$ and M. Warner ${ }^{2}$ \\ ${ }^{1}$ Department of Physics, University of Surrey, Surrey GU2 7XH, United Kingdom \\ ${ }^{2}$ Cavendish Laboratory, University of Cambridge, Madingley Road, Cambridge CB3 OHE, United Kingdom
}

(Received 23 August 2006; revised manuscript received 19 March 2009; published 23 June 2009)

\begin{abstract}
At the $A$ to $C$ transition, smectic elastomers have recently been observed to undergo $\sim 35 \%$ spontaneous shear strains. We first explicitly describe how strains of up to twice this value could be mechanically or electrically induced in $\mathrm{Sm}-C$ elastomers by rotation of the director on a cone around the layer normal with an elastic cost dependent on constraints. Second, for typical sample geometries, we give the various microstructures in Sm- $C$ akin to those seen in nematic elastomers under distortions with constraints. It is possible to give explicit results for the nature of the textures. Chiral $\mathrm{Sm}-C$ elastomers are ferroelectric. We calculate how the polarization could be mechanically reversed by large, hard, or soft strains of the rubber depending upon sample geometry.
\end{abstract}

DOI: 10.1103/PhysRevE.79.061704

PACS number(s): 61.30.Vx, 83.80.Va, 61.41.+e

\section{INTRODUCTION}

One of the most remarkable properties of liquid crystals is their (improper) ferroelectricity when in the chiral smectic- $C$ $\left(\mathrm{Sm}-C^{*}\right)$ phase $[1,2]$. Unlike crystalline ferroelectrics, their polarization is easily switched, for they are liquids, albeit with orientational (nematic) and layered (smectic) order. Thus new types of displays have been based on the fast and ready response of such liquids to electric fields [2-5]. We model the mechanically driven switching of soft-solid analogs $-\mathrm{Sm}-\mathrm{C}^{*}$ elastomers with both orientation and layering where imposed strains can rotate the polarization, $\mathbf{P}$.

Nematic order, about an ordering director $\hat{\mathbf{n}}$, is along the layer normal $\hat{\mathbf{k}}$, in smectic $A(\mathrm{Sm}-A)$ phases. Meyer et al. [1] recognized that when $\hat{\mathbf{n}}$ tilts with respect to $\hat{\mathbf{k}}$ upon entering the $\mathrm{Sm}-C$ phase, then in a chiral system polarization can exist: denote the direction of the projection of $\hat{\mathbf{n}}$ in plane by the unit vector $\hat{\mathbf{c}}$, whence $\hat{\mathbf{k}} \wedge \hat{\mathbf{c}} \rightarrow \hat{\mathbf{p}}$ is an operation that defines a polar unit vector $\hat{\mathbf{p}}$ in a chiral system. Figure 1 shows the $A$ and $C$ phases, with smectically ordered rods rather more sharply confined to layers than is realistic. The spontaneous polarization is a consequence of ferroelectricity that is termed "improper" since the order parameter is related to the tilt [2] rather than the polarization itself as in the case of normal ferroelectrics.

Liquid crystalline (LC) polymers display the same phases as classical materials. The rods, in the sketch Fig. 1, are pendant to main chains which can then be linked to form an LC elastomer. Nematic elastomers suffer large mechanical elongations/contractions on cooling/heating to and from the ordered state. If strains are applied noncoaxially with $\hat{\mathbf{n}}$, director rotation and further sympathetic shears can develop to allow shape change without energy cost in ideal systems and with little energy cost in nonideal systems-so-called soft elasticity $[7,8]$. The magnitude of spontaneous distortion on entering the nematic state sets the scale for the extent of soft deformation when mechanically induced director rotation occurs.

$\mathrm{Sm}-A$ elastomers are not soft because (a) the director is not free to rotate without taking the layers with it and (b) the matrix can only deform while affinely convecting its embed- ded layers with it and respecting the constancy of layer spacing. The smectic layer modulus is much larger than the rubber modulus and hence distortions such as extension along $\hat{\mathbf{k}}$ are very expensive and in most systems they only have small amplitude $[9,10]$ before instabilities arise. Essentially Sm- $A$ rubbers behave two-dimensionally; they stretch and contract in plane only. Their shears have either displacements purely in plane or, if out of plane, they act to rotate the layers. Sm- $A$ rubber elasticity is highly complex and nonlinear.

The same constraints of constancy of layer spacing act on $\mathrm{Sm}-C$ elastomers. The transition $\mathrm{Sm}-A \rightarrow \mathrm{Sm}-C$ is accompanied by a spontaneous shear, $\Lambda$, not trivially related to the molecular tilt angle $\theta[6,11-13]$, as well as an elongation in plane and contraction along the layer normal. The spontaneous shear is large, $\Lambda \sim 0.3-0.4$ in the experiments of [6], Figs. 1(c) and 1(d). We take subsequent distortions with respect to the spontaneously distorted shape of Fig. 1(b) or Fig. $1(\mathrm{~d})$, which is the reference state shown relaxed and without distortion in Fig. 2(a). (A residual shear visible in the Sm- $A$

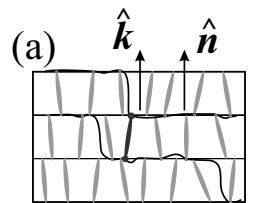

(c)

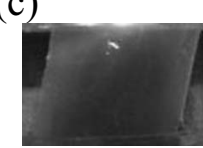

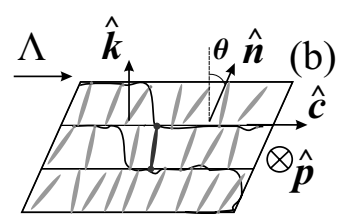

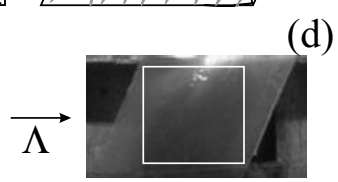

FIG. 1. (a) Smectic- $A$ phase with parallel director $\hat{\mathbf{n}}$ and layer normal $\hat{\mathbf{k}}$. The polymer backbones are for the polymeric case with the rods shown as pendant. A cross-link (dots at either end of the rod) links polymers into the network. (b) Smectic- $C$ phase with $\hat{\mathbf{n}}$ tilted by $\theta$ in a direction defined by the unit vector $\hat{\mathbf{c}}$ in the plane. The third (unit) direction, $\hat{\mathbf{p}}=\hat{\mathbf{k}} \wedge \hat{\mathbf{c}}$, is into the page. It defines the direction of polarization, $\mathbf{P}=P \hat{\mathbf{p}}$. For $\mathrm{Sm}-C$ elastomers, there is a spontaneous shear $\Lambda$ with respect to its $\mathrm{Sm}-A$ parent. Photographs (c) and (d) [6] are of the $A$ to $C$ transition in elastomers. The cut out in (d) is the Sm-C sample in Fig. 2(a) that is to be deformed in this paper. 


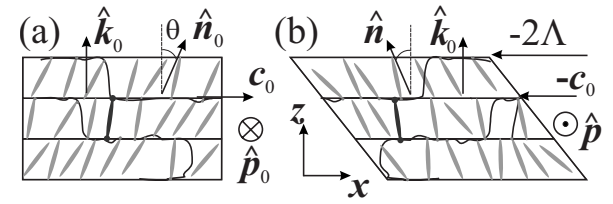

FIG. 2. A block of Sm- $C$ rubber (a) initially undistorted with $\hat{\mathbf{n}}_{0}$ and $\hat{\mathbf{c}}_{0} \equiv \hat{\mathbf{x}}$. The layer normal will be taken to remain along $\hat{\mathbf{z}}$. (b) sheared by $\lambda_{x z}=-2 \Lambda$ with respect to its relaxed state. The in-plane director has reversed, $\hat{\mathbf{c}} \rightarrow-\hat{\mathbf{c}}_{0}$ and thus so has the polarization direction: $\hat{\mathbf{p}}_{0} \rightarrow \hat{\mathbf{p}}=-\hat{\mathbf{p}}_{0}$.

state, Fig. 1(c), is an artifact of the two-step cross-linking method of achieving monodomains.)

Sm- $C$ elastomers can theoretically be soft since the vector $\hat{\mathbf{n}}$ and hence $\hat{\mathbf{c}}$ can rotate about $\hat{\mathbf{k}}$ and in doing so induces shape changes of the body without change in the smectic or rubber elastic energy. Soft elasticity is predicted to be of considerable complexity in $\mathrm{Sm}-C$ elastomers since the layer normal can also rotate $[11,14]$. One can find [14] concrete examples of soft modes where the layer normal remains fixed. We denote the angle of rotation of $\hat{\mathbf{c}}$ about an unchanging layer normal $\hat{\mathbf{k}}_{0}$ by $\phi$. The soft shape changes associated with changing $\phi$ are shears which conclude at $\phi= \pm \pi$, with $\lambda_{x z}=-2 \Lambda$ and all other distortions vanishing. In Fig. 2(b) this final shear corresponds to reversing the spontaneously sheared shape in Fig. 1(b) to its opposite form. The spontaneous simple shear thus has an important role in delineating the extent of softness of imposed deformations in smectic rubbers. Analogously, in experiment and theory of softness in nematic elastomers, the extent is delineated by the extent of spontaneous elongation on entering the nematic phase. For $\phi$ increasing still further, the original undistorted state is eventually regained at $\phi=2 \pi$.

As $\hat{\mathbf{c}}$ rotates about $\hat{\mathbf{k}}$ by $\phi$, then so too does the polarization direction $\hat{\mathbf{p}}$. If it is initially along $\hat{\mathbf{y}}$, then when $\hat{\mathbf{c}}$ has rotated by $\pi, \hat{\mathbf{p}}$ has reversed to $-\hat{\mathbf{y}}$; see Fig. 2(b). Much of this paper is concerned with describing how this reversal of polarization can be achieved by the imposition of shear deformation to the elastomer. The applications of shear generating large electrical changes are obvious and very attractive. With the rotations of $\mathbf{c}$ now defined, we can now give a concrete form of the true order parameter which is an inplane vector $\boldsymbol{\xi}=[(\hat{\mathbf{k}} \wedge \hat{\mathbf{n}}) \wedge \hat{\mathbf{k}}] \wedge \hat{\mathbf{n}}=-(\hat{\mathbf{k}} \wedge \hat{\mathbf{n}})(\hat{\mathbf{k}} \cdot \hat{\mathbf{n}})=\sin \theta \cos \theta$ $(-\sin \phi, \cos \phi)$, see [15], and also [2] using an analogous form found in superconductivity.

Although it is possible to find soft trajectories of deformation that reverse $\hat{\mathbf{p}}$, these will not in general satisfy boundary conditions imposed by, say, rigid electrodes or clamps. One can, in some cases, take combinations of $\pm \phi$ deformations that form a texture that overall satisfies the external constraints, that is, the free energy has been "quasiconvexified" [16], a process well understood in the routes to soft deformation of nematic elastomers [8,17]. We show different textures for $\mathrm{Sm}-\mathrm{C}$ elastomers that depend on the constraints offered by the two sample geometries. The general mathematical problem of how textures in $\mathrm{Sm}-C$ elastomers make possible soft deformations in the presence of constraints has been attacked by Adams et al. [18]. In particular it is possible to find geometries that are soft under tension and hence easier to verify experimentally. Otherwise, $\mathrm{Sm}-\mathrm{C}$ elastomers can deform via nonsoft alternatives that fully satisfy constraints. These will also require the development of textures during deformation and we calculate some of them here. In either case there will be barriers (possibly smaller for soft textures) between the two states of reversed polarization. The choice between soft and nonsoft alternatives will depend upon whether one has sheet or slab geometry-we discuss both choices in Sec. III. In a companion paper to this [19] it is shown that there are two possible types of stripe domains, or textures, of which our textures that do not involve the rotation of smectic layers across the laminate boundary are drawn from one class. For chiral Sm- $C$ elastomers, the internal boundaries are in general charged for the class we are dealing with; the other class has uncharged internal boundaries [19].

Most experiments on $\mathrm{Sm}-A$ and $C$ elastomers have involved mechanical and electrical changes to the tilt angle, e.g., [20]. We are looking at Goldstone modes that instead rotate the director about the layer normal at essentially fixed cone angle of tilt. Experiments on polydomain $\mathrm{Sm}-\mathrm{C}$ elastomers [21] show that this rotation is easily possible and can in fact lead to gross reorganization of the domain structure, even its removal. Being polydomain the deformation was not especially soft, but demonstrated LC mobility in this phase and offers hope for the deformations we predict here. In any event, we hope that the range of deformation paths we describe will urgently stimulate experiments to explore the ferroelectric response of smectic elastomers to shears that oppose the spontaneous distortion that arises on leaving the $A$ state. Our theoretical models will show that the understanding of these systems is completely open.

\section{MODEL FOR NONLINEAR DISTORTIONS OF SMECTIC- $C$ RUBBER}

We adopt a particular model that successfully describes experiments on the nonlinear rubbery and smectic elasticity of Sm- $A$ elastomers, and which has also been applied to $\mathrm{Sm}-C$ elastomers. The underlying nematic rubbery elasticity is subject only to layers moving affinely with the bulk and then only allowing distortions of the bulk that then respect the constancy of layer spacing. Thus material points $\mathbf{R}_{0}$ and layer normals $\hat{\mathbf{k}}_{0}$ in the reference state transform as [10]

$$
\mathbf{R}=\underline{\underline{\lambda}} \cdot \mathbf{R}_{0} \text { and } \hat{\mathbf{k}}=\underline{\underline{\lambda}}^{-T} \cdot \hat{\mathbf{k}}_{0} .
$$

The deformation gradient $\underline{\underline{\lambda}}$ must respect rigid constraints of constancy of volume and of smectic layer spacing. This is because the shear modulus of the isotropic state of the elastomer and for the smectic for deformations not involving layer spacing changes is $\mu \sim 10^{5}-10^{6} \mathrm{~J} / \mathrm{m}^{3}$, nearly 2 orders of magnitude smaller than the smectic layer spacing modulus for elastomers and 4 orders of magnitude smaller than the bulk modulus, and hence deformations avoid layer spacing and volume changes. We discuss in Appendix A how $\mu$ can be estimated by measurements in the Sm state. Thus $\underset{\equiv}{\lambda}$ is rigidly constrained so that $\left|\underline{\lambda}^{-T} \cdot \hat{\mathbf{k}}_{0}\right|=1$ where $-T$ denotes 
transposed inverse. $\operatorname{Det}(\underline{\underline{\lambda}})=1$ expresses constancy of volume. The tilt angle between the director and the layer normal is also rigidly fixed, so $\hat{\mathbf{n}} \cdot \hat{\mathbf{k}}=\cos \theta$.

The free-energy density $f$ and a general soft deformation $\lambda_{s}$ are, respectively,

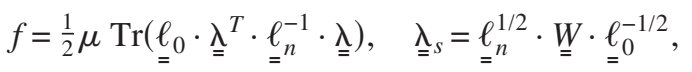

with $\underset{=}{W}$ a general rotation matrix in the nematic case [22]. In smectic elastomers we have a subset of these deformations because of the constraints of layer spacing and tilt angle mentioned above [14]. The shape tensors of the network polymers, $\ell_{0}$ initially and $\ell_{n}$ after deformation (when the director $\hat{\mathbf{n}}_{0} \overline{\bar{h}}$ as possibly rotated to the new direction $\left.\hat{\mathbf{n}}\right)$ are of the form $\ell_{0}=(r-1) \hat{\mathbf{n}}_{0} \hat{\mathbf{n}}_{0}+\underline{\underline{\delta}}$ (and analogously for $\ell_{n}$ ) where $r$ is the anisotropy in the distribution of polymer shapes. These tensors encode information about the direction of order.

For simplicity we take the distribution of backbone chain shapes to be uniaxial. This approximation is discussed in [14] _effects due to rotation of the long axis of the anisotropic distribution are very large and give a first-order description of a system that in reality is certainly to an extent biaxial. In fact there are fundamental reasons why biaxiality generated by the $\mathrm{Sm}-C$ order does not introduce any essentially new elements to the problem-it is locked to the plane spanned by the layer normal and director. As the principal director rotates on a cone about $\hat{\mathbf{k}}$, the other two directions defining a biaxial $\ell_{n}$ are slaves to this motion and retain the same relation to the main director and to the layers. Appendix $\mathrm{B}$ discusses biaxiality in some detail and gives the corresponding soft modes. They are only trivially modified from the uniaxial case and microstructure is modified only slightly, thus justifying the argument above for the neglect of biaxiality in the first approximation.

The minimum free energy is $3 \mu / 2$ which obtains when there is no distortion, $\underline{\underline{\lambda}}=\underline{\underline{\delta}}$ and no director rotation, $\hat{\mathbf{n}}=\hat{\mathbf{n}}_{0}$ and hence $\ell_{=}^{-1}=\ell_{0}^{-1}$, or when deformations are soft, $\underline{\underline{\lambda}}_{s}=\underline{\underline{\lambda}}$.

The spontaneous shear $\lambda_{x z}^{c}$ in this model [12], denoted here by $\Lambda$, is given by

$$
\lambda_{x z}^{\mathrm{c}} \equiv \Lambda=(r-1) / \rho \sin \theta \cos \theta,
$$

where the combination $\rho=r \cos ^{2} \theta+\sin ^{2} \theta \leq r$ will repeatedly follow in the concrete examples of distortions we shall give. The molecular details are thus simply encoded. At fixed temperature, $\theta$ does not change if we also assume that anchoring is rigid, that is, applied strains do not mechanically alter the tilt angle. In considering elastomers with extreme anisotropy of mechanical properties, we are implicitly dealing with smectic elastomers where the layer structure and other details of molecular ordering are on a higher energy scale than rubber elasticity [9]. There may be systems in which tilt is not rigid under imposed strains, a possibility that has been considered theoretically $[11,13,23]$ and experimentally [24]. It is possible that the chain anisotropy $r$ might change with tilt $\theta$ and thus that the $\theta$ dependence is more complicated than appears in Eq. (3). This complication will not concern us for elastomers at fixed temperature, and thus fixed tilt, during mechanical experiments. We are not dealing with smectic elastomers in which layers do not appear to significantly affect mechanical properties, for instance, elastomers where one can induce compression of the smectic layers by applying an in-plane strain [25]. Likewise we are not addressing experiments that (i) use mechanical compression to alter the tilt angle (thereby changing $P$ and hence a route to piezoelectricity) or (ii) apply an electric field, alter tilt, and hence generate strain along the layer normal (an inverse piezoelectric effect) [20,26,27].

We now consider three explicit types of deformation in response to imposed shears that seek to redirect the spontaneous shears observed on the $A$ to $C$ transition. The deformations are treated separately. They have increasing freedom to exercise various sympathetic distortions which serve to progressively soften the elastic energy penalty.

\section{A. Simple nonsoft response to shear}

We are interested in shearing $\mathrm{Sm}-C$ elastomers simply, without rotating or distorting the layers, perhaps by fixing rigid plates to their $x y$ surfaces. First, consider a simple nonsoft deformation with its inverse transpose:

$$
\stackrel{\underline{\lambda}}{=}\left(\begin{array}{ccc}
1 & 0 & \lambda_{x z} \\
0 & 1 & \lambda_{y z} \\
0 & 0 & 1
\end{array}\right), \quad \underline{\lambda}^{-T}=\left(\begin{array}{rrr}
1 & 0 & 0 \\
0 & 1 & 0 \\
-\lambda_{x z} & -\lambda_{y z} & 1
\end{array}\right) .
$$

Trivially $\operatorname{Det}(\underline{\underline{\lambda}})=1$, volume is conserved, and $\underline{\underline{\lambda}}^{-T} \cdot \hat{\mathbf{z}}=\hat{\mathbf{z}}$, the layer normals are not rotated and their separation is unchanged by the action of $\lambda$. The 0 and 1 entries in Eq. (4) ensure that the $x y$ plates do not change shape.

We take an initial $\hat{\mathbf{c}}_{0}=\hat{\mathbf{x}}$ as in Fig. 2(b) and impose a shear $\lambda_{x z}$. The shear $\lambda_{y z}$ is the relaxation expected as $\hat{\mathbf{n}}$ is induced to rotate by $\phi$ about $\hat{\mathbf{k}}$ toward $\hat{\mathbf{y}}$. Inserting $\underset{\equiv}{\lambda}$ into $f$ and minimizing over $\lambda_{y z}$ and $\phi$ gives the optimal free energy and relaxation as a function of $\lambda_{x z}$. The $\underline{\underline{\lambda}}$ of Eq. (4) is not soft: since the director is being rotated, the elongation associated with it is also rotated so one expects extension along $y$, contraction along $x$, and some $y x$ shear in plane (see below where we successively allow these relaxations). There is a cost of constraining diagonal elements to 1 and some shears to 0 , leading to a threshold, $\lambda_{1}$, before $\lambda_{y z}$ relaxation and rotation of $\hat{\mathbf{c}}$ starts. Until then the free energy is hard with a corresponding modulus; see the curvature of the initial part of Fig. 3(a) and full details in Appendix A. For the $r=2, \theta$ $=\pi / 6$ chosen for illustration throughout, the spontaneous shear would be $\Lambda=\sqrt{3} / 7 \simeq 0.246$. When relaxation starts, the free energy is softened considerably since now shape change can be more by rotation of the long axis of the polymer chain distribution than by an expensive distortion of the chains. The shear $\lambda_{y z}$ and rotational $\phi$ relaxation, Fig. 3(b), are both initially singular. Director rotation is directly observable optically. We defer discussing it until we consider sample geometry and the questions of textures and ferroelectric switching. The thresholds are slightly complicated functions of $r$ and $\theta$ that can be found analytically from the free energy. The singular forms arise because both $\pm \lambda_{y z}$ and $\pm \phi$ give the same $\lambda_{x z}$. Thus for instance $\lambda_{x z} \propto \phi^{2}$ or conversely there is a square root singularity $\phi \propto \sqrt{\lambda_{x z}}$, and similarly for $\lambda_{y z}$.

The $y z$ shear reaches a maximum numerically equal to $\Lambda$ at $\phi=\pi / 2$ and $\lambda_{x z}=-\Lambda$ since at this imposed shear $\hat{\mathbf{c}}$ points 


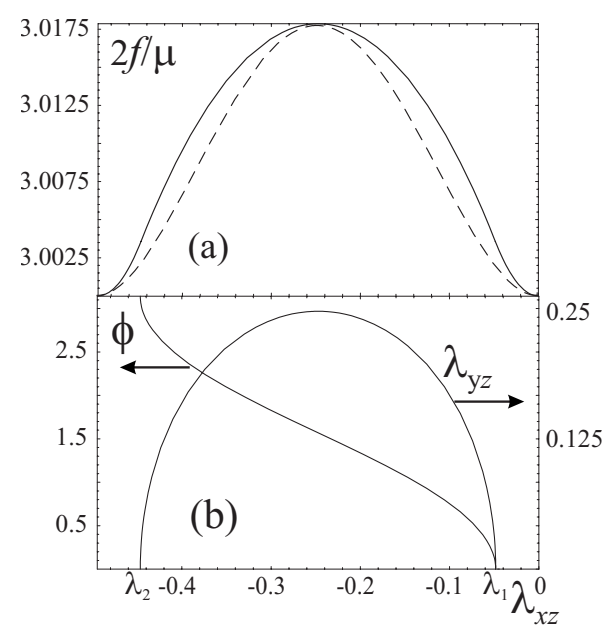

FIG. 3. (a) The elastic free energy, in units of $\frac{1}{2} \mu$, against imposed shear $\lambda_{x z} \leq 0$. The dotted line is energy cost when allowing $y x$ relaxation as well. (b) Rotation $\phi$ of the in-plane director $\hat{\mathbf{c}}$ about the layer normal and the concomitant $y z$ shear relaxation, both starting and concluding at thresholds $\lambda_{1}$ and $\lambda_{2}$, respectively. The anisotropy is $r=2$ and the director tilt is $\theta=\pi / 6$.

along $\hat{\mathbf{y}}$. The anisotropy now along $\hat{\mathbf{y}}$ is analogous to the tilt of the anisotropy along $\hat{\mathbf{x}}$ in Fig. 1(b). The same $y z$ shear develops as was the case with $x z$ shear in going from $\mathrm{Sm}-A$ to $\mathrm{Sm}-\mathrm{C}$ in Fig. 1. But the free energy is instead maximal at this shear. It then declines back to its minimal value $3 \mu / 2$ for an imposed shear of $-2 \Lambda$. The $y z$ relaxation vanishes, and $\phi$ attains $\pi$, in a singular manner at a threshold, $\lambda_{2}$, equivalent to that at small $\lambda_{x z}$, before the $x z$ shear reaches $-2 \Lambda$. In nonideal systems (semisoft elastomers) the free energy would not be at an absolute minimum at $\phi=\pi$. We discuss semisoft response below in considering the role of disproportionation. The singular behavior is seen and expected for analogous distortions involving mechanically induced director rotation in nematic elastomers; see the experiments of Finkelmann et al. [28]. The imposition of shear in a slab geometry (discussed later in Fig. 7) is straightforward. It is also possible in sheets of the form of Figs. 1(c) and 1(d), up to a certain maximum shear where wrinkling onsets. Even corresponding $\mathrm{Sm}-A$ sheets, which are certainly not soft, have been successfully sheared to investigate mechanically induced director tilt into the $C$ form (the mechano-clinic effect) $[24,23]$.

\section{B. Additional nonsoft shear relaxation}

It is possible to find slightly softer deformation trajectories than that illustrated above by including the $y x$ component of deformation. For systems with clamps allowing changes in the shape of the $x y$ section of the elastomer, or for microstructures that we will explore below, this extra freedom might be a way of reducing the elastic energy between the two minimal states at $\phi=0$ and $\phi=\pi$. Thus the deformation gradient and its inverse transpose are, respectively,

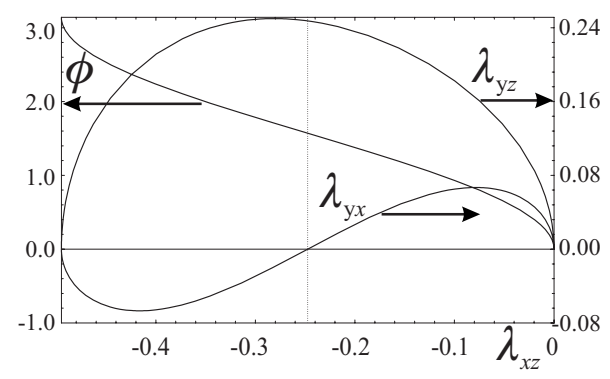

FIG. 4. The $y x$ and $y z$ shears and in-plane director rotation $\phi$ against imposed shear $\lambda_{x z}$ for the softer case where $y x$ relaxation is permitted ( $r$ and $\theta$ as before). The light vertical dotted line is at $\lambda_{x z}=-\Lambda$ to emphasize the asymmetry about the point where the director is transverse; see Appendix C.

$$
\left(\begin{array}{ccc}
1 & 0 & \lambda_{x z} \\
\lambda_{y x} & 1 & \lambda_{y z} \\
0 & 0 & 1
\end{array}\right) \text { and }\left(\begin{array}{ccc}
1 & -\lambda_{y x} & 0 \\
0 & 1 & 0 \\
-\lambda_{x z} & \lambda_{y x} \lambda_{x z}-\lambda_{y z} & 1
\end{array}\right) \text {, }
$$

where, with the aid of the latter, one can confirm that the layer normal has not been rotated nor the layer spacing changed by the imposed deformation. With this greater freedom, the threshold to both director rotation and strain relaxation can be avoided. Thus the free-energy cost is lowered from the previous trajectory; see the dotted curve also plotted in Fig. 3(a) for direct comparison with the energy when less relaxation is permitted. This minimal free energy arises from the optimal deformation gradient $\lambda$, with elements explicitly given below, being put into $f$ of Eq. (2). The same procedure must be followed still further below when an explicit form for a soft deformation is given, but the result for $f$ is then trivial (the unchanging value $3 \mu / 2$ ). Here, the components of the optimal deformation gradient tensor are

$$
\begin{gathered}
\lambda_{x z}=-\frac{r-1}{\rho} \sin \theta \cos \theta(1-\cos \phi) \equiv-\Lambda(1-\cos \phi), \\
\lambda_{y z}=\Lambda\left(1-\frac{r-1}{a^{2} r} \sin ^{2} \theta \cos \phi\right) \sin \phi, \\
\lambda_{y x}=\frac{r-1}{2 a^{2} r} \sin ^{2} \theta \sin 2 \phi,
\end{gathered}
$$

with the combination $a^{2}=\cos ^{2} \phi+(\rho / r) \sin ^{2} \phi \leq 1$. The $y x$ and $y z$ shears plus the accompanying director rotation are plotted in Fig. 4 against the imposed $x z$ shear.

The rotation is straightforwardly $\cos \phi=1+\lambda_{x z} / \Lambda$ from Eq. (6) and must be inserted into Eqs. (7) and (8) to get $\lambda_{y z}\left(\lambda_{x z}\right)$ and $\lambda_{y x}\left(\lambda_{x z}\right)$. The singular rotation initially and finally is just that of the $\cos ^{-1}$ function:

$$
\begin{gathered}
\phi= \pm \sqrt{-2 \lambda_{x z} / \Lambda} \text { for } \lambda_{x z} \lesssim 0, \\
\phi= \pm \sqrt{2\left(2-\lambda_{x z} / \Lambda\right)} \text { for }-2 \Lambda \lesssim \lambda_{x z} .
\end{gathered}
$$

At $\phi=\pi / 2$ one has $\lambda_{x z}=-\Lambda$; the $x z$ section of the sample has gone halfway between the states of Figs. 2(a) and 2(b). 
The new shear, $y x$, is antisymmetric about $\phi=\pi / 2$. It must vanish when $\hat{\mathbf{c}}$ points along $\hat{\mathbf{y}}$ since the natural change in the sample would be to elongate along $\hat{\mathbf{y}}$ (which we as yet suppress, $\left.\lambda_{y y}=1\right)$ and to $y z$ shear. There is no need for inplane shape change, $\lambda_{y x}=0$.

The $y z$ deformation is numerically equal to $\Lambda$ at $\phi=\pi / 2$ as one would expect—when the director has rotated by $\pi / 2$ the $y$ axis has a similar significance to that of the $x$ axis initially, but the energy is now high. This intermediate state $\phi=\pi / 2$ with $\hat{\mathbf{n}}$ in the $y z$ plane and $\hat{\mathbf{c}}=\hat{\mathbf{y}}$ is where there must be maximal $y z$ distortion due to director rotation redirecting the elongated dimension of the sample. Indeed the energy can be seen to be symmetric about $\pi / 2$ (actually seen in the plots against $\lambda_{x z}$ to be symmetric about the value $-\Lambda$ ). However, since we are dealing with large deformations that do not add linearly, the additional shear introduces an apparent asymmetry about $\phi=\pi / 2$ into the form of the $y z$ relaxation. (In Appendix C, we show that this apparent asymmetry, and more asymmetries to be discussed below, are simply consequences of the geometric need to compound rather than add large deformations.) The $y z$ relaxation reaches a numerically maximal value at $\phi=\frac{1}{2} \cos ^{-1}\left\{(r-1) \sin ^{2} \theta /\left[(r-1) \cos ^{2} \theta+r\right.\right.$ $+1]\}$.

The energy maximum offers the same height of barrier between the minimal states as before. With respect to the minimal value of $3 \mu / 2$, the barrier is $2 f_{\mathrm{bar}} / \mu$ $=2(r-1)^{2} \sin ^{4} \theta /\{r[r+(r-1) \cos 2 \theta]\}$. As in all expressions involving the energy cost on rotation or shear, it scales as $(r-1)$, that is, it vanishes on isotropy $(r=1)$. Otherwise, the energy is somewhat reduced, see the dotted curve in Fig. $3(\mathrm{a})$, but at the expense of a more complex system of sympathetic shears. We now explore a final shear scenario to lower the free energy of distortion still further to its minimal value.

\section{Soft deformations}

If the rubber has total freedom to shear and distort as the director rotates, then in ideal systems there is zero accompanying rubber elastic cost. The mechanism arises because the distribution of chains is accommodated by the changing shape of the body without the distribution's distortion and thus with no decrease in the entropy or modification of the liquid crystal order. Such distortions are well known in nematic elastomers [7] deep into the nonlinear regime. They have been explored theoretically in $\mathrm{Sm}-C$ elastomers $[11,13,14]$ where the constraint of layers must be rigidly observed. A soft deformation gradient (and its inverse transpose), which leaves the layer normal unrotated and the layer spacing unchanged, is

$$
\begin{gathered}
\underline{\underline{\lambda}}=\left(\begin{array}{ccc}
\lambda_{x x} & 0 & \lambda_{x z} \\
\lambda_{y x} & \lambda_{y y} & \lambda_{y z} \\
0 & 0 & 1
\end{array}\right), \\
\stackrel{\lambda}{=}^{-T}=\left(\begin{array}{ccc}
\lambda_{y y} & -\lambda_{y x} & 0 \\
0 & \lambda_{x x} & 0 \\
-\lambda_{y y} \lambda_{x z} & \lambda_{y x} \lambda_{x z}-\lambda_{x x} \lambda_{y z} & 1
\end{array}\right),
\end{gathered}
$$

where $\lambda_{z z}=\lambda_{x x} \lambda_{y y}=1$ from incompressibility, $\operatorname{Det}(\underline{\underline{\lambda}})=1$. The elements of the tensor are [14]

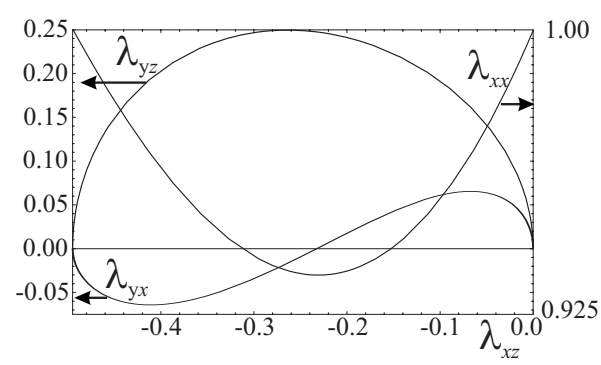

FIG. 5. The $y x$ and $y z$ shears and the $x x$ contraction against imposed shear $\lambda_{x z}$ for soft deformations ( $r$ and $\theta$ as before).

$$
\begin{gathered}
\lambda_{x x}=1 / \lambda_{y y}=a(\phi), \\
\lambda_{x z}=\Lambda[-a(\phi)+\cos \phi], \\
\lambda_{y x}=\frac{r-1}{2 r a} \sin ^{2} \theta \sin 2 \phi, \\
\lambda_{y z}=\Lambda\left(\sin \phi-\frac{r-1}{2 r a} \sin ^{2} \theta \sin 2 \phi\right),
\end{gathered}
$$

with $a^{2}=\cos ^{2} \phi+(\rho / r) \sin ^{2} \phi$ which was introduced below Eq. (8), and $\Lambda$ given in Eq. (3). A factor $\left(1-\frac{\rho}{r}\right)$ appearing in the expression derived in [14] has been replaced by the equivalent $(r-1) \sin ^{2} \theta / r$. The same types of shears as in Eq. (5) enter, but we allow the elongations and contractions $\lambda_{x x}$ and $\lambda_{y y}$ to adjust to the changing natural length in the $x$ and $y$ directions as the polymer chain distribution anisotropy is rotated by $\phi$. It is this final element, plus the concomitant further changes to the shears, that allows the deformation to be soft. Figure 5 shows the $x x$ contraction and the $y x$ and $y z$ shears against the imposed $x z$ shear.

In Fig. 6 we show snapshots of an initial cube deforming under a soft $\lambda$ as the $\hat{\mathbf{c}}$ director advances (right to left) through $\phi=0, \pm \pi / 2, \pm 2 \pi / 3$, and $\pm \pi$. It starts from Fig. 2(a) and ends in (b), but we are viewing it from along the $z$ axis rather than along the $y$ axis. Such a monodomain response, either along the $+\phi$ or $-\phi$ route, may not conform to macroscopic constraints, for instance, those that suppress $\lambda_{y z}$ shear. Much of the remainder of this paper is about adding $\pm \phi$ distortions in microtextures to conform to constraints.

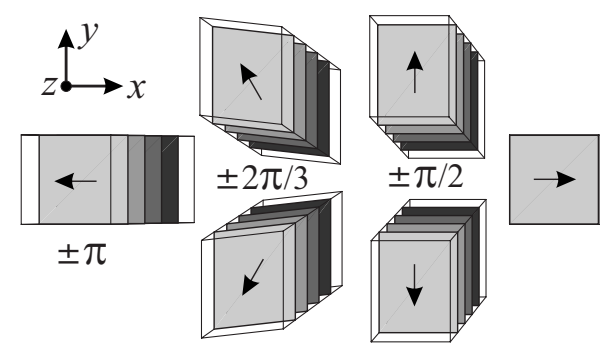

FIG. 6. Soft deformations of a cube of Sm- $C$ rubber with $r=8$ and $\theta=\pi / 6$ (as before) viewed along the smectic layer normal. The c vectors and the corresponding $\phi$ are shown. 


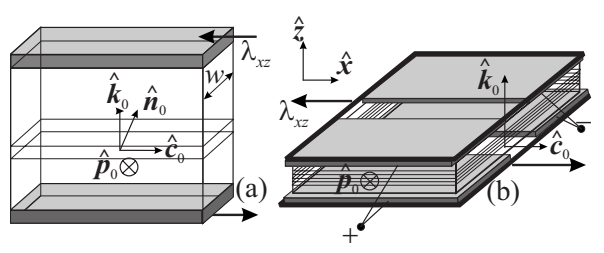

FIG. 7. (a) Sheet and (b) slab geometries for $\mathrm{Sm}-\mathrm{C}$ elastomers. Rigid clamps (shaded) are shown on the $x y$ sample surfaces. $\lambda_{x z}$ shear displacements are indicated by arrows. A few smectic planes are indicated in each case, with normals $\hat{\mathbf{k}}_{0}$. polarization $\mathbf{P}$ in the $y$ direction (of extent $w$ ) emerges from the $x z$ sample surfaces. Lines of electric flux in the slab case are captured by enveloping the small $x z$ surfaces by overhanging, split $x y$ electrodes, their charge being identified by \pm .

The rotation $\phi$ is of the singular form as before, with slight modifications that are apparent on comparing the soft $\lambda_{x z}(\phi)$, Eq. (14), with the corresponding $x z$ deformation gradient, Eq. (6), in the hard case with $y x$ relaxation. Explicitly, $\phi\left(\lambda_{x z}\right)$ follows from Eq. (14):

$$
\phi=\cos ^{-1}\left\{\frac{r}{\rho}\left[\sqrt{\left(\frac{\rho}{r}\right)^{2}+\left(1-\frac{\rho}{r}\right)\left(\frac{\lambda_{x z}}{\Lambda}\right)^{2}}+\frac{\lambda_{x z}}{\Lambda}\right]\right\}
$$

with singular rotation initially (and analogously finally):

$$
\phi \sim \pm \sqrt{\frac{2 r}{\rho} \lambda_{x z} / \Lambda} \text { for } \lambda_{x z} \lesssim 0 .
$$

Note that $\lambda_{x z}$ is no longer $-\Lambda$ when the $\hat{\mathbf{c}}$ director is along $\hat{\mathbf{y}}$, that is, $\phi=\pi / 2$, but is instead

$$
\begin{aligned}
\lambda_{x z}=-\Lambda \sqrt{\rho / r} & =-\Lambda \sqrt{1-\frac{r-1}{r} \sin \theta \cos \theta} \\
& \equiv-\Lambda \lambda_{x x}(\phi=\pi / 2),
\end{aligned}
$$

a numerically smaller value than before. Figures 2(a) and 2(b) correspond to shears of 0 and $-2 \Lambda$ and when the system has $\phi=\pi / 2$ it is geometrically halfway between. The apparent inconsistency, because the shear is not $-\Lambda$, is again a consequence of nonlinearity; see Appendix B. However, when the $\hat{\mathbf{c}}$ director is transverse, there is, as expected, no in-plane shape change, $\lambda_{y x}=0$, as can be seen in Eq. (15) for this $\phi$. The $y z$ shear can be seen to be $\Lambda$ at $\phi=\pi / 2$, but since $\lambda_{x z}>-\Lambda$ there, then $\lambda_{y z}\left(\lambda_{x z}\right)$ is asymmetric about $-\Lambda$ as in the previous subsection, which is because of the geometrical nonlinearity of finite deformations.

\section{SAMPLE GEOMETRY AND TEXTURED RESPONSE}

The form of deformation in response to an imposed shear $\lambda_{x z}$ or director rotation $\phi$ (driven perhaps electrically) will depend on what components of $\lambda$ are inhibited by geometrical constraints. We now discuss different sample geometries. Hiraoka et al. [6] created monodomains and observed large spontaneous shears on the $\mathrm{Sm}-A \rightarrow \mathrm{Sm}-C$ transition in a sheet geometry, Fig. 7(a), where the $y$ dimension of the sample is small. Alternatively smectic actuation, albeit from electro- clinic effects in $\mathrm{Sm}-A^{*}$ elastomers where tilt and shear are purely electrically driven, has been achieved [20] with small shears in the slab geometry of Fig. 7(b), a geometry that is also highly interesting for Sm- $C$ elastomers. In each case the sample is effectively held by rigid clamps-tape overlapping the sheet sample, and by glass plates in the slab case. We expect changes in the polarization, $\mathbf{P}$ to be along $\hat{\mathbf{y}}$ and hence suitable flexible electrodes would be needed for detection of charges on the $x z$ faces for the sheet sample. In the slab case, rigid $x y$ electrodes under the $x y$ plates are suggested for detecting $x z$ surface charges by capturing the lines of $\mathbf{E}$ emerging from the sample surfaces with $\mathbf{y}$ as normal.

Near the rigid clamps we have $\lambda_{x x}=1$ and $\lambda_{y y}=1$ in the slab case. For sheets this constraint is over a small fraction of the sample. The variation in $\lambda_{x x}$, say, in the $z$ direction away from the boundaries to more favorable bulk values is slow, thus generating (by compatibility) minor $\lambda_{x z}$ additions to the deformation. Under these circumstances one could expect elastically the sheet deformations to be of the soft form Eq. (11) where the additional freedom gives a much reduced energy cost. By contrast, the slab geometry imposes a constant $\lambda_{x x}=1$ throughout because the sample is thin in the $z$ dimension. Slabs then could at best have the distortion Eq. (5) where the diagonal elements are all 1. Analogously in slabs, rigid $x y$ plates would suppress in their close vicinity any $\lambda_{y x}$ shear. It is possible that in the bulk of slabs $\lambda_{y x}$ is suppressed, though $z$ variation of $\lambda_{y x}$ generates through compatibility $\lambda_{y z}$ which we are already considering and it is possible that even for slabs, in the bulk one must consider $\lambda_{y x} \neq 0$.

In both sheets and slabs, we have seen how imposing $\lambda_{x z}$ induces macroscopic translations in the $y$ direction resulting from the accompanying $\lambda_{y x}$ and $\lambda_{y z}$ shears. Such shears drastically reduce the energy. However, when the rigid bounding plates are constrained to move only in the $x$ direction, there is a conflict with the sympathetic shears that can be overcome by the development of microstructure or textures of bands of $y z$ shears of alternating sign, corresponding to rotations of alternating sign, $\pm \phi$, but with the same $\lambda_{x z}$. At the macroscopic level the $\lambda_{i j}$ components that are odd in $\phi$ cancel with each other, and those even in $\phi$ add. Thus a body, with $\pm \phi$ rotations and thus also \pm shears in equal proportions, as a whole has a deformation gradient of the form

$$
\underline{\lambda}_{\mathrm{mac}}=\left(\begin{array}{ccc}
\lambda_{x x} & 0 & \lambda_{x z} \\
0 & \lambda_{y y} & 0 \\
0 & 0 & 1
\end{array}\right)
$$

even though locally $\lambda_{y x}( \pm \phi) \neq 0$ and $\lambda_{y z}( \pm \phi) \neq 0$. (The $x x$ deformation gradient might vanish, $\lambda_{x x}=1$, as discussed above for slabs.) The alternation ensures no macroscopic, constraint-violating, $\hat{\mathbf{y}}$ displacements develop across the sample. Such textures will be required for all three deformations we have explored, soft or nonsoft. The development of microstructure is analogous to that in the soft deformations of nematic elastomers where the underlying soft deformations are observed, but where necessary in textures to allow the underlying soft response to occur [29]. Texture is not the cause of softness, but a symptom that arises from softness in the presence of constraints. 


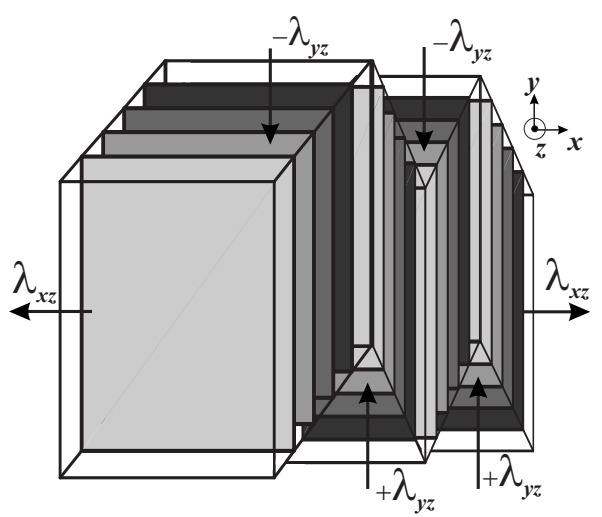

FIG. 8. A slice through a solid deforming with a textured version of Eq. (4) for $\underline{\underline{\lambda}}$. Alternating $\pm \lambda_{y z}$ shears lead to no overall macroscopic $\lambda_{y z}$ but to the desired imposed $\lambda_{x z}$.

The reader can see a concrete example of how to construct $\lambda_{\text {mac }}$ by putting, say, the $\phi=\pi / 2$ body of Fig. 6 on top of that with $\phi=-\pi / 2$. (At this special $\phi$, this picture is close to that in Fig. 8 below.) Looking at the position of the overall top $x y$ surface with respect to that of the bottom, it is clear that there has been a $x z$ shear, but that overall there is no $y$ relative displacement and thus overall no $\lambda_{y z}$. What is important is that the bodies are placed on top of each other, rather than in any other configuration, a question of the compatibility of textures to which we turn below. With the above $\underline{\lambda}_{\text {mac }}$ the energy will have been reduced (to zero in the ideal soft case) and yet overall the body has conformed to boundary conditions that are inconsistent with the underlying deformations (4), (5), and (11). The development of textures to achieve soft response in the face of incompatible boundary conditions quasiconvexifies the free energy, a process well understood in Martensite [16] and in nematic elastomers $[8,17]$. It is important in the response of the constrained samples that we are studying here.

We now derive the full form of the textures that arise for the two geometries that do not rotate the smectic layer normals. We limit ourselves to such textures since then the $x y$ sample faces can then retain their orientation as deformation proceeds. Recall that the textures we have described, with unrotating smectic layers, are only part of one of a possible two families of textures in Sm-C elastomers [19].

\section{A. Forms of textures}

Neighboring laminates in a texture with deformations $\underline{\underline{\lambda}}_{ \pm \phi}$ that are in contact through a common surface must suffer deformations that are rank-one connected [16], otherwise there is a geometric inconsistency between the specification of translations of their interfacial elements by $\underline{\underline{\lambda}}+\phi_{+}$and $\underline{\underline{\lambda}}_{-\phi}$, that is:

$$
\underline{\lambda}_{+\phi}-\underline{\underline{\lambda}}_{-\phi}=\mathbf{a} \otimes \mathbf{S}_{\phi}=2\left(\begin{array}{ccc}
0 & 0 & 0 \\
\lambda_{y x} & 0 & \lambda_{y z} \\
0 & 0 & 0
\end{array}\right)
$$

since $\lambda_{y x}(\phi)$ and $\lambda_{y z}(\phi)$ are both odd about $\phi=0$ whereas $\lambda_{x z}(\phi), \lambda_{x x}(\phi)$, and $\lambda_{y y}(\phi)$ are even. Here $\mathbf{a}$ is a vector in a laminate's surface in the target state and $\mathbf{s}_{\phi}$ is the surface normal of a laminate back in the reference state, that is the normal to the boundary of a region that will, after rotations of $\pm \phi$, transform into a laminate. It is straightforward to see that (uniquely)

$$
\mathbf{a}=(0,1,0) \text { and } \mathbf{s}_{\phi} \propto\left(\lambda_{y x}, 0, \lambda_{y z}\right) .
$$

As the textures evolve with $\phi$, their laminates have normals in the $z x$ plane and, if they rotate at all with changing $\phi$, it is about the $y$ axis. The texture normal in the target space, $\mathbf{s}_{\phi}^{\prime}$, is given by the usual transformation for normals of planes embedded in an elastic solid, $\mathbf{s}_{\phi}^{\prime}=\lambda_{\phi}^{-T} \cdot \mathbf{s}_{\phi}$.

\section{Textures without elongations and in-plane shears}

For the simple deformation Eq. (4) with $\lambda_{y x}=0$, the normal is $\mathbf{s}_{\phi}=\mathbf{s}_{\phi}^{\prime} \propto\left(0,0, \lambda_{y z}\right)$. Thus the textures and the smectic layers share the unchanging normal $\mathbf{s}^{\prime}=\hat{\mathbf{k}}_{0}=\hat{\mathbf{z}}$, Fig. 8. Successive $x y$ slices of the solid suffer alternating $\lambda_{y z}$ transverse shears. This pattern is a possibility for slab geometries, but less likely for sheets where a soft texture is a possibility we examine below. There is no question of polarization charges residing on the internal surfaces of this texture since $\mathbf{P}$ rotates in the plane of the laminates and never passes through the internal interfaces. This texture is a special case of the generally charged family.

\section{Textures with in-plane shears}

For both the other choices of deformation, the normal $\mathbf{s}_{\phi}^{\prime}$ of the transformed laminates has a universal form regardless of softness, that is, it holds for both classes (B) and (C) of Sec. II:

$$
\begin{aligned}
\mathbf{s}_{\phi}^{\prime} & \propto\left(\lambda_{y y} \lambda_{y x}, 0, \lambda_{y z}-\lambda_{y y} \lambda_{y x} \lambda_{x z}\right) \\
& \rightarrow(\sin \theta \cos \phi, 0, \cos \theta) .
\end{aligned}
$$

Therefore the current (target space) laminate normal $\mathbf{s}_{\phi}^{\prime}$ has an angle, $\chi^{\prime}$, to the $z$ axis:

$$
\chi_{\phi}^{\prime}=\tan ^{-1}[\cos \phi \tan \theta] .
$$

Contrast this case with the more trivial case above where the laminate normal was along $z$, that is, along the layer normal. The need for more subtle laminates can be seen in Fig. 6 where, except for $\phi=0, \pm \pi / 2, \pm \pi$, the top surface is sheared away from its initial square shape with shears $\pm \lambda_{y x} \neq 0$ of alternating sign. Now stacking $\pm \phi$ bodies on top of each other to eliminate a macroscopic $\lambda_{y z}$ shear is impossible since the faces do not match, unless the laminates are tilted with respect to the layers.

For different rotation angles $\phi$ of the $\hat{\mathbf{c}}$ director around $\hat{\mathbf{k}}_{0}$, the normal $\mathbf{s}_{\phi}$ or $\mathbf{s}_{\phi}^{\prime}$ to the laminates takes differing rotation angles about the $\hat{\mathbf{y}}$ axis, Fig. 9. For $\phi=\pi / 2$, both $\chi=0$ and $\chi^{\prime}=0$, that is $\mathbf{s}_{\phi}$ and $\mathbf{s}_{\phi}^{\prime}$ are along $\hat{\mathbf{k}}_{0}$ and the laminates are aligned with the smectic planes. Again Fig. 6 makes this clear-at this $\phi$ there is no $y x$ shear and no impediment to simply stacking the \pm regions as simple laminates. To explain why rank-one connectedness requires laminates rotated 


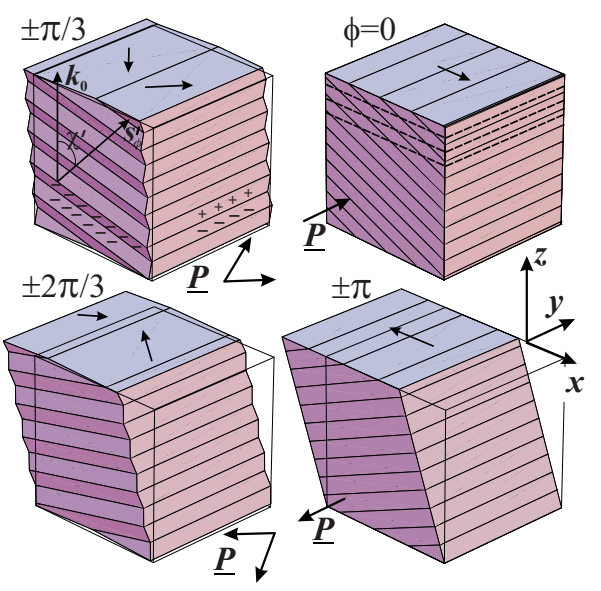

FIG. 9. (Color online) An initially cubic sample deforming softly with laminates of $y x$ and $y z$ shears of alternating sign and with $x z$ shear advancing from 0 to $-2 \Lambda$ in steps of $\pm \pi / 3$ in director rotation $\phi$ about the $z$ axis (marked on figures; see also the $\mathbf{c}$ relevant to each laminate as it emerges at the top face of the sample). The smectic layers (a few dotted on the $\phi=0$ snapshot) are unrotating as $\phi$ changes and retain their normal $\hat{\mathbf{k}}_{0}=\mathbf{z}$. The alternating shear deformations lead to no corresponding macroscopic shears whereas the imposed $\lambda_{x z}$ does. The laminate normal $\mathbf{s}_{\phi}^{\prime}$ in the deformed (target) state is shown for the example of $\phi=\pi / 3$. It starts and finishes parallel to $\hat{\mathbf{n}}$ but in general makes an angle $\chi$ with the layer normal $\hat{\mathbf{k}}_{0}$ given in the text. The deformations away from the initial shape (light reference frame given in $\phi \neq 0$ pictures) reveal the slight contraction $\lambda_{x x}$ along $x$ and a compensatory lengthening along $y$, see, e.g., $\phi=\pi / 3$, for $\phi \neq 0, \pm \pi$. The $\pm \pi / 2$ case is like Fig. 8, that is, with $\chi=0$, but with $\lambda_{x x}, \lambda_{y y} \neq 1$.

with respect to the smectic layers, Fig. 9, we transform $\lambda$ from the $(x, y, z)$ frame to one based upon $\mathbf{s}$, that is, $(\perp, y, s)$ by rotating both the target and reference states, thus $\underset{\equiv}{\lambda} \rightarrow \underline{\underline{\lambda}}^{\prime}$ $=\underline{\underline{W}} \cdot \underline{\underline{\lambda}} \cdot \underline{\underline{U}}^{T}$ by $\chi$ about $y$. The target and reference state rotations are $\underline{\underline{W}}$ and $\underline{\underline{U}}$, respectively. The rotations have the effect of mixing the $\lambda_{y x}$ and $\lambda_{y z}$ shears to give a $\lambda_{y \perp}=\cos \chi \lambda_{y x}$ $-\sin \chi \lambda_{y z}$, which vanishes by definition of $\mathbf{s}$, Eq. (22). The laminate planes at this orientation no longer suffer the inplane shears that would make them impossible to match across their interfaces.

Our analysis of soft modes and nonsoft modes with $y x$ relaxation has been based upon the free rotation of the normal of the system of laminates about the $y$ axis. It is possible that the motion of the laminate surfaces, through the assembly of smectic layers that is stationary, is in fact pinned. A rubber is liquidlike at the molecular level, accounting for the extreme extensibility of rubbers and, for instance, their motionally narrowed NMR lines. It is known that laminates evolve and rotate easily in nematic elastomers responding at low-energy cost [29]. Experiments on polydomains [21] show that it is likely that textures in smectic- $C$ elastomers can evolve under imposed strains and that low-energy deformations are thus possible as we propose. Experiment is vital to determine whether pinning is active or not.

Another uncertainty is the energy cost of laminate walls. The directors on opposite sides of a laminate surface are rotated to $\pm \phi$. At $\phi=\pi / 2$ the layers and laminates share a common normal and laminate surfaces are a $\pi$ twist-bend

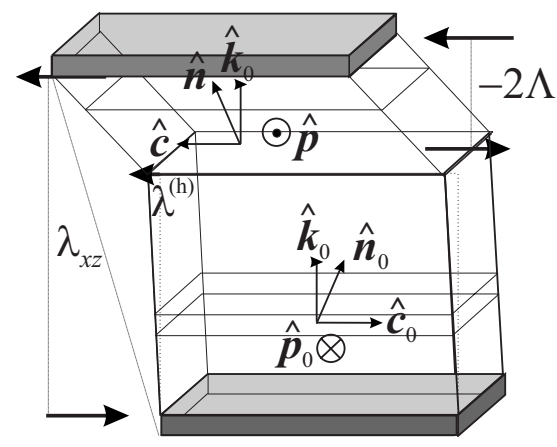

FIG. 10. A sample deforming by disproportionation. The macroscopic $\lambda_{x z}$ shear displacements are indicated by heavy arrows and consists of a fraction (lower) of weakly sheared $\lambda^{(h)}$ sample and the complementary (upper) fraction distorted by $-2 \Lambda$. The macroscopic shear is $\lambda_{x z}$.

wall. At other values of $\phi$, the laminates and layers are oblique to each other and the wall is more complicated. In any event there is a cost to walls that demands calculation when experiment in the two geometries has been carried out. This energy cost and any competing one associated with the texture will determine, as in nematic elastomers, the ultimate length scale of the structure. A simple qualitative argument yields the usual scaling form of the texture periodicity: consider a simple case where the texture is $x y$ slabs of thickness $\mathcal{L}$ and with surface energy $\gamma$ per unit area. Let $w_{i}$ be the extent of the sample in the $i$ th direction. Then there are $w_{z} / \mathcal{L}$ slabs each of energy $\gamma w_{x} w_{y}$. Looking ahead to our analysis of polarization, there are strips of surface charge alternating with period $\mathcal{L}$ on the $y z$ ends of the sample; see Fig. 9. These charges give fields extending outside the sample an $x$ distance $\sim \mathcal{L}$ (by Poisson's law they decay exponentially in the $x$ direction with decay length $\mathcal{L}$ ). The fields scale with $P \cos \phi$ and thus the field energy outside the sample is of the form $\gamma_{p} \mathcal{L} w_{y} w_{z}$ where $\gamma_{p} \sim \epsilon_{0} P^{2}$. The overall energy has competing $\mathcal{L}$ terms. Minimization over $\mathcal{L}$ gives $\mathcal{L} \sim \sqrt{w_{x} \gamma / \gamma_{p}}$. More complicated calculations are needed for greater precision.

\section{Disproportionation}

One perhaps important possible form of texture remains, a simple disproportionation that might occur in both slab and sheet geometries. Analogous disproportionations have been proposed in nematic elastomers [30], though in practice it seems textures are selected. The top portion of the sample is $x z$ sheared to its maximum soft extent, $-2 \Lambda$, with rotation $\phi=\pi$ and the bottom portion is weakly $x z$ sheared by $\lambda^{(h)}$ with unrotated director; see Fig. 10. The bottom must be sheared so that it has a shear stress to match that in the upper part. Any discontinuity of this stress on traversing the interface between the two parts would lead to a net body force.

If the material were ideally soft then the shear stress in distorting the upper portion would be zero and the lower portion would thus remain unsheared. The two states would be separated by a $\pi$ twist-bend wall. It is possible that a sample might deform this way if rotations of textures are pinned and if the energy cost of laminate interfaces discussed 
above is too high. Only one interface is required for this mode of deformation and that interface's normal does not rotate with increasing over all $\lambda_{x z}$. Simple geometrical considerations determine the volume fraction $\Phi$ of the sample that is transformed into the $\phi=\pi, \lambda_{x z}=-2 \Lambda$ state. For this soft case it is $\Phi=\lambda_{x z} /(-2 \Lambda)$. Thus the interface migrates down the sample of Fig. 10 as $\lambda_{x z}$ increases. A particularly simple ferroelectric response then arises, as we see in the next section, and no internal surfaces are charged and thereby add to the energetic cost.

In practice there are small, so-called "semisoft" elastic energies to be paid in nonideal elastomers where softness would otherwise hold. Our current smectic elastomer model is that of nematic elastomer distortion energy under smectic layer spacing constraints. In nematic elastomers nonideality is known to introduce a threshold before essentially soft deformation associated with director rotation onsets. There is a plateau where the stress increases only slowly until the director fully rotates during which the sympathetic director rotation and the elongations, contractions, and shears are identical in form to the ideal case [8]. For nonideal Sm- $C$ elastomers disproportionating, the commons stress in the two portions determined by the upper portion is that characteristic of the end of the semisoft deformation plateau, that is, the maximum value of low stresses before hard deformation starts. To develop a matching stress, the bottom portion would then have to $x z$ shear as well, without any sympathetic deformations (since they would not be rank-one connected to the state of the upper portion). This would be a hard deformation and thus of small amplitude $\lambda^{(h)}$, as sketched in Fig. 10 . Now the volume fraction $\Phi$ that is transformed into the $\phi=\pi$ state is $\Phi=\left(\lambda_{x z}-\lambda^{(h)}\right) /\left(-2 \Lambda+\lambda^{(h)}\right)$ and the interface would migrate down the sample as $\lambda_{x z} \rightarrow-2 \Lambda$.

However, because of nonideality in practice such simple disproportionating response would not seem probable-the alternative is to form textures in the way we have outlined above. The stress due to semisoftness is always lower in the textured case than the disproportionated case except at the end of the rotation of the director when they are equal. In the textured case one would initially find that the semisoft stress is small because $\pm \phi \sim 0$. However in the disproportionation case the director jumps from $\phi=0$ to $\pi$ in part of the sample, which has a much larger associated semisoft stress because of the larger director rotation. Textures would therefore develop at lower energy cost than the higher stress alternative of developing the same $\lambda_{x z}$ through disproportionation. The favoring of textures over disproportionation would be lost as samples approach extreme softness and the cost of internal boundaries gets relatively high.

\section{B. Alternative route to observing microstructures}

We have proposed experimentally observing textures in $\mathrm{Sm}-C$ elastomers from thin samples in the sheet geometry, and polarization microscopy. Classical buckling instabilities are eventually observed in shear experiments of this sort and may obscure the textures [23,24]. Alternatively, a tensile geometry provides a simpler experiment to reveal soft deformations in Sm- $C$ elastomers and their associated microstructures.

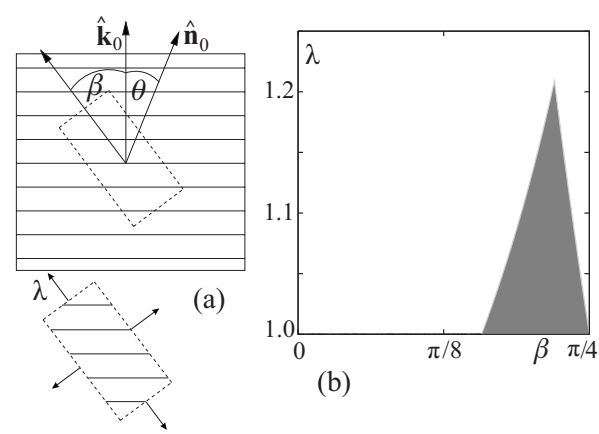

FIG. 11. (a) A sample with the layer normal at an angle $\beta$ to the long direction is cut out of a monodomain Sm-C. (b) The shaded region is that of elongations $\lambda$ for tension angles $\beta$ for which the sample remains soft (for $\theta=22.5^{\circ}$ and $r=2$ ).

A sample of elastomer with an arbitrary angle $\beta$ between the proposed elongation axis and the layer normal can be prepared by cutting from a larger sample as illustrated in Fig. 11(a). When this sample is stretched then it will deform softly provided it can form appropriate microstructures [18]. The extent of the soft deformation is illustrated in Fig. 11(b). For small $\beta$ there are no microstructures that permit a soft deformation, but for an interval of $\beta$ from above $\pi / 8$ until close to $\pi / 4$, the sample can deform softly.

\section{FERROELECTRIC RESPONSE}

When a polarization $\mathbf{P}$ changes on passing through a surface, by Gauss's theorem a surface charge density $\sigma=$ $-\Delta P_{\perp}$ develops that is equal to the change in the normal component of $\mathbf{P}$ from one side to the other. Thus at the $x z$ external surfaces of both sample geometries, $\sigma^{(x z)}=P \cos \phi$ $\equiv P_{y}$; see for instance the explicit decoration of $x z$ surfaces with charges in the $\pm \pi / 3$ snapshots in Fig. 9. We now relate $\sigma^{(x z)}$ to the imposed deformation $\lambda_{x z}$ in the three cases of deformation:

(i) The nonsoft deformation with a threshold has singular director rotation against imposed $\lambda_{x z}$ because it too is essentially of the $\cos ^{-1}$ form that subsequent deformation modes are also shown to be. Between $\lambda_{1}$ and $\lambda_{2}$, the variation is almost indistinguishable from $\cos \phi=\left(2 \lambda_{x z}-\lambda_{1}-\lambda_{2}\right) /\left(\lambda_{1}\right.$ $\left.-\lambda_{2}\right)$, where $\lambda_{1}$ and $\lambda_{2}$ are the thresholds to director rotation that arise in this constrained case. Thus the surface charge evolves linearly with $\lambda_{x z}$ :

$$
\sigma^{(x z)}=-P\left(2 \lambda_{x z}-\lambda_{1}-\lambda_{2}\right) /\left(\lambda_{1}-\lambda_{2}\right) .
$$

(ii) For the nonsoft deformation without a threshold, Eq. (5), one sees directly from Eq. (6) that the $x z$ surface charge density is

$$
\sigma^{(x z)}\left(\lambda_{x z}\right)=-P\left(1+\lambda_{x z} / \Lambda\right) .
$$

Recall that the imposed shear $\lambda_{x z}$ varies from 0 to $-2 \Lambda$ and thus this surface charge reverses linearly with the applied shear deformation. It would not change any further, were the shear to be increased beyond $-2 \Lambda$.

(iii) When deformation is soft, the connection between $\cos \phi$ and $\lambda_{x z}$ is slightly more complicated, despite being 
linear at the start and end of the rotation. Equation (17) for $\cos \phi \equiv \sigma / P$ gives the explicit shear dependence of the $x z$ surface charge density:

$$
\sigma_{\mathrm{soft}}^{(x z)}=-P \frac{r}{\rho}\left[\sqrt{\left(\frac{\rho}{r}\right)^{2}+\left(1-\frac{\rho}{r}\right)\left(\frac{\lambda_{x z}}{\Lambda}\right)^{2}}+\frac{\lambda_{x z}}{\Lambda}\right] .
$$

\section{A. Charging of laminate surfaces; overall electrostatic energy and mechanical stability}

For the nontrivial textures with the laminate normals not along the layer normals, the normal component of the polarization can reverse at the internal surfaces separating laminates in the texture. One must then consider the energies of the resulting internal surface charge distributions in both the cases of sheets and slabs. The laminate normals $\mathbf{s}_{\phi}^{\prime}$ rotate about $y$ in the $x z$ plane. The $y$ component of $\mathbf{P}$, giving rise to the $x z$ external surface charges discussed above and pictured in Figs. 2 and 7 and the $\pm \pi / 3$ part of Fig. 9, does not contribute to the internal sheets of charge. The internal charges arise rather from the reversal of the $P_{x}$ component at the laminate surfaces, that is, $\sigma_{\text {lam }} \propto P \hat{\mathbf{x}} \cdot \mathbf{s}_{\phi}^{\prime}$; see Fig. 9. The internal surface charge density is therefore

$$
\frac{\sigma^{\mathrm{lam}}}{P}= \pm \sin \phi \sin \chi= \pm \frac{1}{2} \frac{\tan \theta \sin 2 \phi}{\left(1+\tan ^{2} \theta \cos ^{2} \phi\right)^{1 / 2}} .
$$

One must ask then whether these charges have a significant energetic effect.

In the sheet geometry relatively little of the otherwise uniform field generated by the $x z$ sheets of external surface charge leaks out of the sample. The $y$-internal electric displacement in the sample is $D_{(y)}^{\text {int }}=\sigma^{(x z)}=-P \cos \phi$. If the textures are fine, the electric displacement between internal surfaces of alternating charge is also not lost, and is $D_{\mathbf{s}_{\phi}^{\prime}}^{\text {lam }}$ $=P \sin \phi \sin \chi$, from Eq. (29). This $D$ is in the $\pm \mathbf{s}_{\phi}^{\prime}$ directions.

Finally the component of $P_{x}$ that intersects the external $y z$ surfaces generates $\sigma^{(y z)}$ external surface charge densities; see the charges shown on the $y z$ surfaces of the $\pm \hat{\mathbf{p}} / 3$ snapshot of Fig. 9. These are $\sigma^{(y z)}=-P \sin \phi \cos \chi$ and change sign between the $y z$ ends of neighboring laminates as $\phi \rightarrow-\phi$. The charges generate small effective internal fields because the $y z$ surfaces are small, are widely separated in the sheet geometry and, in any event, are oppositely charged in stripes, so we can ignore their energetic effect.

At the exceptional points $\phi= \pm \pi / 2$, the internal fields $E_{\text {int }}^{y}=0$ and $E_{\text {lam }}=0$, the latter because the laminate normals are along the layer normals $z$. All the surface charges are confined to the $y z$ external surfaces which we have just argued generate an ignorable internal field.

The overall electrostatic energy density, $f_{\mathrm{el}}$, is thus essentially only from internal fields generated by charges appearing on the internal surfaces of textures. These fields depend in part on the anisotropic dielectric tensor of the elastomer which we represent for simplicity, see Appendix D, by a single averaged value $\varepsilon$ :

$$
\begin{aligned}
f_{\mathrm{el}} & =\frac{1}{2} \frac{P^{2}}{\varepsilon \varepsilon_{0}}\left\{\cos ^{2} \phi+\sin ^{2} \phi \sin ^{2} \chi\right\} \\
& =\frac{1}{2} \frac{P^{2}}{\varepsilon \varepsilon_{0}} \cos ^{2} \phi /\left(\cos ^{2} \theta+\sin ^{2} \theta \cos ^{2} \phi\right) .
\end{aligned}
$$

There is a weak $\chi$ dependence in $1 / \varepsilon$ that we estimate in Appendix D and argue that we can neglect in the estimates below.

At first sight this energy is perhaps alarming. $f_{\mathrm{el}}$ is maximal, $f_{\mathrm{el}}=P^{2} /\left(2 \epsilon \epsilon_{0}\right)$ at $\phi=0, \pm \pi$ and is minimal, $f_{\mathrm{el}}=0$, at $\phi= \pm \pi / 2$. For soft deformations, where there is no elastic cost, the director should spontaneously rotate to $\phi= \pm \pi / 2$ and the sample should spontaneously shear to $-\Lambda$. It is likely however that the $x z$ external surfaces, that are initially charged, attract counterions from their surroundings and are neutralized. There is accordingly no electrostatic cost at $\phi$ $=0$ (and finally at $\phi= \pm \pi$ ) since no internal field $E_{\mathrm{int}}^{y}$ is generated. The only internal fields are those between successive (internal) laminate surfaces (unless these too are neutralized by internal conduction processes). The electrostatic energy density is then simply

$$
f_{\mathrm{el}}^{\prime}=\frac{P^{2}}{\epsilon \epsilon_{0}} \frac{\sin ^{2} 2 \phi \sin ^{2} \theta}{\cos ^{2} \theta+\sin ^{2} \theta \cos ^{2} \phi} .
$$

There is thus no electrostatic cost at $\phi=0, \pm \pi / 2, \pm \pi$. All these points are local minima of the free energy and the cost in deviating from these points means that spontaneous rotation is avoided. It does suggest however that there is a complex energy associated with the path between $\lambda_{x z}=0$ and $-2 \Lambda$ for softly deforming elastomers. Should the deformation be of the hard type with $\lambda_{y x} \neq 0$, which demands complex textures as in the soft case, then the elastic energy has to be added to the electrostatic energy $f_{\mathrm{el}}^{\prime}$, the resultant of which depends on the relative scale of rubber elastic and ferroelectric energies.

Values of $P$ for smectic $C^{*}$ systems are in the range $10^{-5}-10^{-4} \mathrm{C} / \mathrm{m}^{2}[8]$, with saturation values of $P$ for some polymers used for $\mathrm{Sm}-C^{*}$ networks being reported as large as $2 \times 10^{-3} \mathrm{C} / \mathrm{m}^{2}$ [26]. One must compare the resulting energy density, $\sim \frac{1}{2} P^{2} / \epsilon_{0}$, with elastic energy densities in the problem which are $\frac{1}{2} \mu 10^{-2}$, taking the strain energy at the maximal (barrier) value, $10^{-2}$ in units of $\mu / 2$; see Fig. 3(a). For $\mu \sim 10^{5} \mathrm{~J} / \mathrm{m}^{3}$ this puts the ratio of the electrostatic to elastic energies in the range $10^{-2}$ to 1 - under some circumstances electrostatics may be important, making the need for experimental results still greater to discern the different mechanisms that will be selected by these competitive energies. It is possible that one might have complicated free energies of deformation that result from the sum of the elastic and electrostatic influences.

In the slab geometry hard deformations should result because of the rigid constraints imposed near the surface of the clamps. Consequently the laminate normals are along the layer normals and hence always perpendicular to the polarization that rotates about the $z$ axis as $\phi$ evolves. Accordingly, no internal sheets of charge develop for intermediate imposed shears. 
The charges on the $x z$ and $y z$ external surfaces give rise to fields that largely leak out of the sample and hence again we ignore their contribution to the energy. The leaking field lines from the $x z$ surfaces can be captured by overhanging electrodes as in Fig. 7(b) and hence the surface charge, and $P$, can be measured.

As mentioned above, we have described only one of a possible two families of textures in Sm-C elastomers. The other family of textures can be shown [19] not to have charges accumulating on their internal surfaces. They are not necessarily applicable to the constraints of unrotating smectic layers that we have considered.

\section{B. Electrically driven actuation?}

The inverse response when applying a potential to elastomers in the two geometries in order to generate a shear strain also needs quantifying. Elastic barriers to deformation still exist, but now one is coupling external potentials to the charge layers that exist on the free surfaces, that is, achieving an energy change per unit $x z$ area of sample of $\frac{1}{2} \sigma V$ on achieving $\sigma=0$ as a result of applying a voltage $V$ across the sample and mechanically switching as far as the maximum in the elastic energy barrier (where $\phi=\pi / 2$ ). For the favorable case of the sheet, taking $V=10^{3} \mathrm{~V}$ and thickness $w=1 \mathrm{~mm}$, the relative energy densities (electrical to elastic) are $V P /\left(w \mu 10^{-2}\right) \sim 10^{-2}-1$ considering the range of values of $P$ given above. The reason is that internal electrical fields that set the scale of energies above are $E_{\text {int }} \sim 10^{7} \mathrm{~V} / \mathrm{m}$, much larger than those typically able to be applied here. One concludes that electromechanical actuation at very large shear strains $(\sim 1)$ will require the sheet geometry with large fields.

\section{Experimental observations}

The internal fields, which we argue could play a significant role in fixing the mechanical stability of these elastomers in their textured state, are perhaps open to direct observation that might help determine what rotations are accompanying shears. These intense fields are given, see Appendix D, by $\mathbf{E}=\frac{1}{\varepsilon_{0}} \epsilon^{-1} \cdot \mathbf{D}$ with $\mathbf{D}=P_{s^{\prime}} \mathbf{s}_{\phi}^{\prime}$ the electric displacement generated by the internal surface charges and directed along the laminate normals. Thus $E_{i}=\frac{1}{\epsilon_{0}} \epsilon_{i s^{\prime}}^{-1} D_{s^{\prime}}$. The field is not purely along the laminate normal because this direction is not a principal direction of the dielectric tensor. Guest species with, for instance, characteristic absorption or fluorescence could be aligned with the field rather than with, say, the director or smectic layer normal. In particular a distinction between the field and director directions emerges as soon as rotation starts and would be an independent check of the development of microstructure. Absorption or florescence probes suggested above are invariant under $\mathbf{s}_{\phi}^{\prime} \rightarrow-\mathbf{s}_{\phi}^{\prime}$ and thus would not find the alternating texture structure nugatory. It is likely that the choice of geometry, slab or sheet, will yield very different results.

\section{CONCLUSIONS}

The deformation path taken by a smectic- $C$ elastomer in having the direction of its spontaneous polarization mechani- cally or electrically rotated and eventually reversed is calculated for slab and sheet geometries. We are concerned with the Goldstone mode of director rotation about layer normals on a cone of fixed angle, rather than mechanical and electrical induction of a change in cone angle. Experiments on the alignment of polydomain smectic elastomers [21] by external stress demonstrate that director rotation is easily possible and is an important aspect of deformation, as it is also in nematic elastomers. To conform to constraints while deforming at low elastic energy, it is proposed that textures develop. The laminates of these textures are at nontrivial angles to the layer system and rotate with respect to the layers as the strain develops. Different possible textures result according to geometry, the constraints at surfaces, the elastic shear energy, the role of semisoftness, and the charging of internal texture surfaces when they cut the polarization. One extreme case of geometric disproportionation is discussed and found to be unlikely. However, important determinants such as the relative roles of polarization and elastic cost are still most open and experiments on large deformations are urgently required.

\section{ACKNOWLEDGMENTS}

We are grateful to Professor K. Bhattacharya for pointing out the necessity to examine the effect of charges on internal surfaces that led to the analysis of Sec. IV A. John Biggins made important suggestions about disproportionation and on the character of $\mathrm{Sm}-\mathrm{C}$ stripe domains generally. Hiraoka et al. gave us permission to use the photographs of Fig. 1.

\section{APPENDIX A: ENERGY SCALE $\mu$ FOR DEFORMATIONS}

We model smectic elastomers as essentially nematic elastomers with strong constraints of layering. Thus $\mu$ that scales the underlying rubber elastic energy would be the shear modulus for the rubber, were it able to enter its isotropic state. More usefully one can relate it to certain shear or extensional moduli in the $\mathrm{Sm}-C$ elastomeric state. Elongation along the $\hat{\mathbf{c}}_{0} \equiv \hat{\mathbf{x}}$ direction, $\lambda_{x x}>1$, or shear with displacement in the positive $x$ direction, $\lambda_{x z}>0$, both do not rotate the $\hat{\mathbf{c}}$ director, provided the layer normal $\hat{\mathbf{k}}_{0}$ is fixed (for instance by clamps or rigid plates). We are assuming rigid Sm order-for instance in-plane elongations are accompanied by $\lambda_{y y}=1 / \lambda_{x x}$ since contraction along $z$ is forbidden (it would alter the layer spacing) and these deformations are two dimensional as in the case of Sm- $A$ elastomers undergoing similar strains [9] and which have been modeled in these terms [10]. Without rotations and other shears, the free energy cannot drastically reduce, as in the rest of this investigation. The free energies of such distortions are

$$
\begin{aligned}
f\left(\lambda_{x z} \geq 0\right)= & \frac{1}{2} \mu\left(3+\frac{1}{4 r}[r+1+(r-1) \cos 2 \theta]^{2} \lambda_{x z}^{2}\right), \\
f\left(\lambda_{x x} \geq 1\right)= & \frac{1}{2} \mu\left(\frac{\left(\lambda_{x x}-1\right)^{2}}{8 r}\left[r^{2}+1-(r-1)^{2} \cos 4 \theta\right]\right. \\
& \left.+\frac{1}{4 \lambda_{x x}^{2}}\left[4+3 \lambda_{x x}^{2}+2 \lambda_{x x}^{3}+3 \lambda_{x x}^{4}\right]\right),
\end{aligned}
$$

with corresponding small strain moduli: 


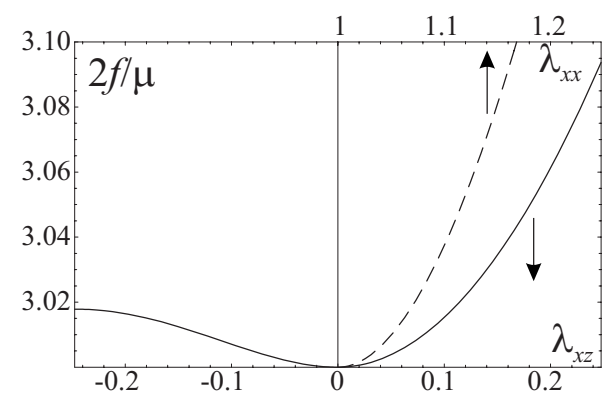

FIG. 12. Energy of deformation against both positive (hard) and negative (softer) shear $\lambda_{x z}$, and against extensions $\lambda_{x x} \geq 1$ (dotted curve; $\lambda_{x x}$ upper scale) up to strains of $\pm \Lambda= \pm 0.246$, for the values of $r$ and $\theta$ adopted in the text. Note how much lower the energy of distortion is if sympathetic relaxations and director rotation are permitted.

$$
\begin{gathered}
k_{x z}=\frac{\mu}{4 r}[r+1+(r-1) \cos 2 \theta]^{2}, \\
k_{x x}=\frac{\mu}{8 r}\left[1+30 r+r^{2}-(r-1)^{2} \cos 4 \theta\right] .
\end{gathered}
$$

A sense of how much easier deformation is with relaxation can be seen in Fig. 12.

The moduli, along with the spontaneous distortion (or limits to softer deformation and completion of charge switching) and conoscopy (for $\theta$ ), give experimental insight into $\mu$, $r-1$, and $\cos \theta$.

\section{APPENDIX B: THE EFFECT OF BIAXIALITY}

Because layers break the cylindrical symmetry of the underlying nematiclike distribution of chain shapes when it is tilted with respect to the layers, the shape tensor is biaxial; see Fig. 13. For Sm-C or Sm- $C^{*}$ systems the layer normal $\hat{\mathbf{k}}$ and the director $\hat{\mathbf{n}}^{\prime}$ define another plane, with normal $\hat{\mathbf{n}}^{\prime} \wedge \hat{\mathbf{k}}$. (We now denote the rod director by $\hat{\mathbf{n}}^{\prime}$.) Two of the principal directions of the polymer shape tensor are in the plane spanned by $\hat{\mathbf{k}}$ and $\hat{\mathbf{n}}^{\prime}$ and are denoted by $\hat{\mathbf{n}}$ and $\mathbf{p}_{1}$, and are respectively associated with the longest (typically) and shortest extents of $\ell$. The third axis, $\mathbf{p}_{2}$, is along $\hat{\mathbf{n}}^{\prime} \wedge \hat{\mathbf{k}}$. [One can see this from ${ }^{\prime}$ constructing $\ell$ from available double-headed vectors in terms of $\hat{\mathbf{n}}^{\prime} \otimes \hat{\mathbf{n}}^{\prime}, \hat{\mathbf{k}} \otimes \hat{\mathbf{k}},\left(\hat{\mathbf{n}}^{\prime} \cdot \hat{\mathbf{k}}\right)\left(\hat{\mathbf{n}}^{\prime} \otimes \hat{\mathbf{k}}+\hat{\mathbf{k}} \otimes \hat{\mathbf{n}}^{\prime}\right)$ and $\underline{\underline{\delta}}$ for $\mathrm{Sm}-C$, and additionally $\left(\hat{\mathbf{n}}^{\prime} \wedge \hat{\mathbf{k}}\right) \otimes\left(\hat{\mathbf{n}}^{\prime} \wedge \hat{\mathbf{k}}\right)$ for

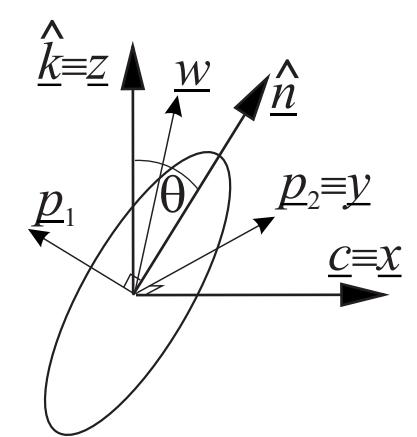

FIG. 13. The principal director, $\hat{\mathbf{n}}$, of the shape tensor is tilted with respect to the layer normal, $\hat{\mathbf{k}}$, by an angle $\theta$. Thereby the other two principal directions, $\mathbf{p}_{1}$ and $\mathbf{p}_{2}$, are distinguished to give a biaxial distribution and thus also a biaxial shape tensor $\underline{\underline{\ell}}$, here seen in section.

Sm- $C^{*}$.] In a biaxial environment $\hat{\mathbf{n}}$, now defined in terms of the shape distribution, need not be exactly parallel to the rod director $\hat{\mathbf{n}}^{\prime}$. It might have an angular offset, constant during rotations, which is not important for rubber elasticity which depends on $\ell_{n}$.

We reduce the step lengths by that in the $\mathbf{p}_{1}$ direction so that $\ell$ is

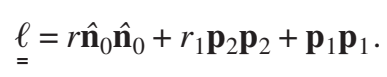

Typically, since layers can squash chains down, one might expect the secondary aspect ratio $r_{1}>1$. Assuming that layers convect with distortions affinely, that is, layer normals evolve like $\hat{\mathbf{k}}=\underline{\lambda}^{-T} \cdot \hat{\mathbf{k}}_{0}$, and that layer spacing rigidly resists change, that is, $|\hat{\mathbf{k}}|=1$, then one can show [14] that soft modes leaving the layer normal unrotated are of the general form $\lambda_{\underline{s}}=\ell_{\hat{\mathbf{n}}}^{1 / 2} \cdot W_{\hat{\mathbf{k}}}(\phi) \cdot \underline{W}_{\mathbf{w}_{0}}(\xi) \cdot \ell_{\hat{\mathbf{n}}_{0}}^{-1 / 2}$. As in the remainder of the paper, the director is rotated by an angle $\phi$ about the unchanging layer normal $\hat{\mathbf{k}}$. The other rotation by $\xi$ is about an axis $\mathbf{w}_{0}$ which is an auxiliary vector in the problem defined as $\mathbf{w}_{0}=\ell_{=}^{1 / 2} \cdot \hat{\mathbf{k}}$. One must be careful to also rotate $\mathbf{p}_{1}$ so that it remains locked to the plane of $\hat{\mathbf{k}}$ and $\hat{\mathbf{n}}$. This has the consequence of making any shape changes resulting from the biaxial aspects of $\ell$ slaves of the principal shape changes resulting from $\hat{\mathbf{n}}$ and thus do not add essentially to the problem. The resulting $\underline{\underline{\lambda}}_{\text {soft }}$ is

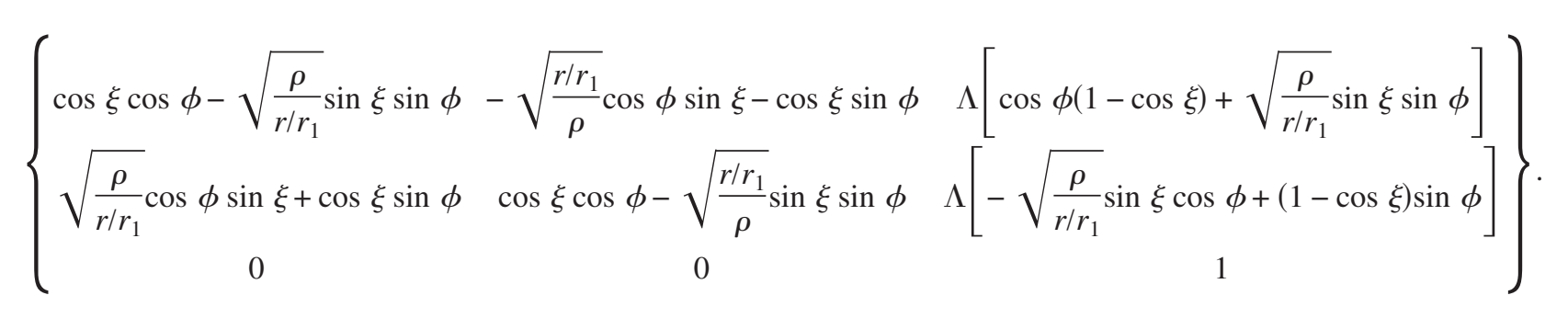


The constant $\rho \equiv \mathbf{w}_{0} \cdot \mathbf{w}_{0}=1+(r-1) \cos ^{2} \theta$ is unchanged from before [see after Eq. (3)]. The abbreviation $\Lambda$ introduced in Eq. (3) is also employed for brevity. The matrix is only modified by some of the appearances of $r$ being rescaled by $r_{1}$. The two vanishing elements ensure that the tensor $\lambda^{-T}$ has no effect on the layer normal and arises from the choice of

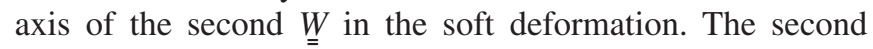
angle $\xi$ can be chosen so that the $\lambda_{x y}$ component is zero. The choice is slightly modified from the uniaxial case: $\tan \xi=$ $-\sqrt{\rho /\left(r / r_{1}\right)} \tan \phi$. With this choice the elements of the deformation gradient tensor, Eqs. (13)-(16), are modified to

$$
\begin{gathered}
\lambda_{y x}=\frac{\sin 2 \phi}{2 a}\left[1-\rho /\left(r / r_{1}\right)\right], \\
\lambda_{y z}=\Lambda\left\{\sin \phi-\frac{\sin 2 \phi}{2 a}\left[1-\rho /\left(r / r_{1}\right)\right]\right\},
\end{gathered}
$$

with a slightly modified $a^{2}=\cos ^{2} \phi+\left[\rho /\left(r / r_{1}\right)\right] \sin ^{2} \phi$ appearing here and also in the otherwise unaltered expressions for $\lambda_{x x}, \lambda_{y y}$, and $\lambda_{x z}$. Unlike in the text, we have not replaced the factor $\left(1-\frac{\rho}{r}\right)$ appearing in the expression derived in [14] by $(r-1) \sin ^{2} \theta / r$ since now there is rescaling of $r$ in the denominator by $r_{1}$. The shears that occur sympathetically to the director rotation $\phi$ are seen to be only slightly modified by biaxiality. Recall from Eq. (23) that the target space laminate normal $\mathbf{s}_{\phi}^{\prime}$ has an angle, $\chi^{\prime}$, to the $z$ axis given in terms of these elements. It will thus be slightly modified in direction. Connection (25) now becomes

$$
\tan \chi_{\phi}^{\prime}=\cos \phi \tan \theta-\frac{r}{r-1} \frac{r_{1}-1}{r_{1}} \frac{\cos \phi}{\sin \theta \cos \theta} .
$$

The first term is as before. The second term vanishes in the Sm- $A$ limit of $\theta=0$ since $r_{1} \rightarrow 1$. A good qualitative understanding of Sm-C laminate-dominated deformations is thus obtained by the neglect of biaxiality since no new element is introduced by it.

\section{APPENDIX C: NONLINEARITY AND APPARENT ASYMMETRIES IN DEFORMATIONS}

The nonsoft deformation (5) leads to a $\lambda_{y z}$ apparently asymmetric about $\phi=\pi / 2$, or equivalently $\lambda_{x z}=-\Lambda$. (A dotted vertical line in Fig. 4 at $\lambda_{x z}=-\Lambda$ makes clear the lack of symmetry.) Manifestly when $\hat{\mathbf{c}}$ has been rotated to $\hat{\mathbf{y}}$, the $y z$ shear is geometrically maximal and should reverse to zero as $\phi \rightarrow \pi$ just as it advanced while $\phi \rightarrow \pi / 2$. We demonstrate that the failure of Eq. (7) and Fig. 4 to exhibit symmetry about $\lambda_{x z}=-\Lambda$ is only apparent. It is a consequence of geometrical nonlinearity (the need to compound rather than add finite deformations).

Consider deformed states of the solid at $\phi=\pi / 2+\zeta$. One can compound deformations,

$$
\underline{\lambda}_{\phi}=\underline{\lambda}_{\zeta}^{\prime} \cdot \underline{\lambda}_{\pi / 2}
$$

where $\lambda_{\phi}$ and $\lambda_{\pi / 2}$ (unprimed tensors) are with respect to the original reference state, $\mathbf{X}^{0}$ say, while $\lambda_{\zeta}^{\prime}$ is a deformation gradient with respect to the reference state $\mathbf{X}^{\prime}$ resulting from the deformation $\lambda_{=/ 2}$, that is, $\mathbf{X}^{\prime}=\underline{\lambda}_{\pi / 2} \cdot \mathbf{X}^{0}$. Using Eqs. (6)-(8) with $\phi=\pi / 2$ for $\lambda_{\pi / 2}$, and adopting form (5) again for $\lambda_{\zeta}^{\prime}$, one obtains, respectively,

$$
\left(\begin{array}{ccc}
1 & 0 & -\Lambda \\
0 & 1 & \Lambda \\
0 & 0 & 1
\end{array}\right) \text { and }\left(\begin{array}{ccc}
1 & 0 & \lambda_{x z}^{\prime} \\
\lambda_{y x}^{\prime} & 1 & \lambda_{y z}^{\prime} \\
0 & 0 & 1
\end{array}\right)
$$

In $\lambda_{\zeta}^{\prime}$ one has $\lambda_{x z}^{\prime}$ odd in $\zeta$ since it continues to decrease below $-\Lambda$. Likewise $\lambda_{y x}^{\prime}$ is also odd since it has returned to zero at $\zeta=0$ and must now become negative for the second half of the range of $\phi$. The shear $\lambda_{y z}^{\prime}$ is even since it reached its maximum of $\Lambda$ when the director points transversely and then must decline again. The roles of $x$ and $y$ in shears involving $z$ have thus been interchanged in this reference state for $\lambda^{\prime}$ with $\hat{\mathbf{c}}$ initially along $\hat{\mathbf{y}}$, just as it was initially along $x$ for the reference state of $\underline{\underline{\lambda}}$. Important for this argument is that rotations of $\pm \zeta$ are physically equivalent.

Consider the difference $\underline{\underline{\Delta \lambda}}$ in the $\underline{\lambda}_{\pi / 2+\zeta}$ and $\underline{\lambda}_{\pi / 2-\zeta}$

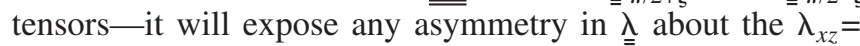
$-\Lambda$ point.

$$
\begin{gathered}
\underline{\underline{\Delta \lambda}}=\left(\underline{\underline{\underline{\lambda}}}_{\zeta}^{\prime}-\underline{\underline{\lambda}}_{-\zeta}^{\prime}\right) \cdot \lambda_{\pi / 2} \\
=2\left(\begin{array}{ccc}
0 & 0 & \lambda_{x z}^{\prime}(\zeta) \\
\lambda_{y x}^{\prime}(\zeta) & 0 & 0 \\
0 & 0 & 0
\end{array}\right) \cdot\left(\begin{array}{ccc}
1 & 0 & -\Lambda \\
0 & 1 & \Lambda \\
0 & 0 & 1
\end{array}\right), \\
\Delta \lambda_{y z}=-2 \lambda_{y x}^{\prime}(\zeta) \Lambda \neq 0 .
\end{gathered}
$$

The extra, $y x$, relaxation permitted in Eq. (5) has created an asymmetry in $\lambda_{y z}$ about $\pi / 2$ that is not a reflection of the true symmetry about $\phi=\pi / 2$ that exists in $y z$ shear. We used in the $\lambda_{y z}^{\prime}$ this very (odd) symmetry with respect to the $\phi$ $=\pi / 2$ state.

Likewise in Eq. (11) for soft deformations, allowing further additional elements in $\lambda$ introduces further geometrical nonlinearity. The energy in the softer case however remains symmetric about $\lambda_{x z}=-\Lambda$, as expected from the equivalence of states with differing $\phi$; see the dotted curve in Fig. 3(a). The discussion around Eq. (19) gives an explicit value for the $x z$ shear at $\phi=\pi / 2$ which is shown not to be $-\Lambda$, that is not simply half the shear associated with a $\pi$ rotation of the $\hat{\mathbf{c}}$ director; geometric nonlinearity is responsible.

\section{APPENDIX D: ELECTROSTATIC ENERGY FROM INTERNAL FIELDS}

The laminate surface charge densities are $\sigma^{\mathrm{lam}}=-\Delta P_{s^{\prime}}$ which generate the displacement fields $D_{s^{\prime}}=\sigma^{\mathrm{lam}}$. The electric-field energy density is $\frac{1}{2} \mathbf{D} \cdot \mathbf{E}$ with $\mathbf{E}=\frac{1}{\epsilon_{0}} \underline{\epsilon^{-1}} \cdot \mathbf{D}$. From this expression and given that $\mathbf{D}$ is in the $\mathbf{s}_{\phi}^{\prime}$ direction, it is clear for the energy that we need the element $\left(\underline{\epsilon}^{-1}\right)_{s^{\prime} s^{\prime}}$ that in Eq. (30) and thereafter we have denoted by $1 / \epsilon$ :

$$
f_{\mathrm{el}}=\frac{1}{2 \epsilon_{0}} D_{s^{\prime}} \epsilon_{s^{\prime} s^{\prime}}^{-1} D_{s^{\prime}}
$$

(no summation over $s^{\prime}$ ). 
One can make various estimates of the relevant element. One assumption would be to take $\underline{\underline{\epsilon}}$ to be uniaxial about the director $\hat{\mathbf{n}}$. We have already made a similar assumption with the $\ell$ tensor. However the $\mathrm{Sm}-C^{*}$ phase is anything but uniaxial in its electrical properties-it has ferroelectric ordering along the in-plane direction perpendicular to c. However, one could take the view that the ferroelectric ordering is sterically driven and remains rigid in the face of internal fields. If one then is examining dielectric effects that result from the polarization of the liquid crystal in the more conventional sense, then perhaps the uniaxial assumption is not so strange as may seem at first sight. In this event, one can characterize $\underline{\underline{\epsilon}}$ by $\epsilon_{\|}$along $\hat{\mathbf{n}}$ and $\epsilon_{\perp}$ in the directions perpendicular to $\hat{\mathbf{n}}$. In this frame, one can write $\underline{\underline{\epsilon}}$ simply and then extract the element required for Eq. (D1):

$$
\underline{\underline{\epsilon}}^{-1}=\left(\frac{1}{\epsilon_{\|}}-\frac{1}{\epsilon_{\perp}}\right) \hat{\mathbf{n}} \hat{\mathbf{n}}+\frac{1}{\epsilon_{\perp}} \underline{\underline{\delta}}
$$

$$
\left(\underline{\underline{\epsilon}}^{-1}\right)_{s^{\prime} s^{\prime}}=\frac{1}{\epsilon_{\perp}}+\left(\frac{1}{\epsilon_{\|}}-\frac{1}{\epsilon_{\perp}}\right)\left(\hat{\mathbf{n}} \cdot \mathbf{s}^{\prime}\right)^{2},
$$

with $\hat{\mathbf{n}} \cdot \mathbf{s}^{\prime}=\cos \theta \cos \chi+\sin \theta \sin \chi \cos \phi$. Rearrangement with the aid of Eq. (25) gives $\hat{\mathbf{n}} \cdot \mathbf{s}^{\prime}=\cos \theta \sec \chi$. This expression and further use of Eq. (25) reduces Eq. (D3) to

$$
\left(\underline{\underline{\epsilon}}^{-1}\right)_{s^{\prime} s^{\prime}}=\frac{1}{\epsilon_{\perp}}+\left(\frac{1}{\epsilon_{\|}}-\frac{1}{\epsilon_{\perp}}\right)\left(\cos ^{2} \theta+\sin ^{2} \theta \cos ^{2} \phi\right) .
$$

Thus the element denoted by $1 / \varepsilon$ in our energy expressions has some $\phi$ dependence, but it is weak compared with the dependence we concentrate on in the energy.
[1] R. B. Meyer, L. Liebert, L. Strzelecki, and P. Keller, J. Phys. (France) Lett. 36, 69 (1975).

[2] S. T. Lagerwall, Ferroelectric and Antiferroelectric Liquid Crystals (Wiley-VCH, Weinheim, 1999).

[3] N. Clark and S. Lagerwall, Appl. Phys. Lett. 36, 899 (1980).

[4] I. Muševič, R. Blinc, and B. Žeks, The Physics of Ferroelectric and Antiferroelectric Liquid Crystals (World Scientific, Singapore, 2000).

[5] S. Lagerwall, Ferroelectrics 301, 15 (2004).

[6] K. Hiraoka, W. Sagano, T. Nose, and H. Finkelmann, Macromolecules 38, 7352 (2005).

[7] I. Kundler and H. Finkelmann, Macromol. Rapid Commun. 16, 679 (1995).

[8] M. Warner and E. M. Terentjev, Liquid Crystal Elastomers (Oxford University Press, Oxford, 2007).

[9] E. Nishikawa and H. Finkelmann, Macromol. Chem. Phys. 200, 312 (1999).

[10] J. M. Adams and M. Warner, Phys. Rev. E 71, 021708 (2005).

[11] O. Stenull and T. C. Lubensky, Phys. Rev. Lett. 94, 018304 (2005).

[12] J. M. Adams and M. Warner, Phys. Rev. E 73, 031706 (2006).

[13] O. Stenull and T. C. Lubensky, Phys. Rev. E 74, 051709 (2006).

[14] J. M. Adams and M. Warner, Phys. Rev. E 72, 011703 (2005).

[15] S. A. Pikin, Structural Transformations in Liquid Crystals (Gordon \& Breach, New York, 1991).

[16] K. Bhattacharya, Microstructure of Martensite (Oxford University Press, Oxford, 2003).
[17] S. Conti, A. DeSimone, and G. Dolzmann, J. Mech. Phys. Solids 50, 1431 (2002).

[18] J. M. Adams, S. Conti, and A. DeSimone, Contin. Mech. Thermodyn. 18, 319 (2007).

[19] J. S. Biggins and K. Bhattacharya Phys. Rev. E 79, 061705 (2009).

[20] W. Lehmann, H. Skupin, C. Tolksdorf, E. Gebhard, R. Zentel, P. Krüger, M. Lösche, and F. Kremer, Nature (London) $4 \mathbf{4 1 0}$, 447 (2001).

[21] A. Sanchez-Ferrer and H. Finkelmann, Macromolecules 41, 970 (2008).

[22] P. D. Olmsted, J. Phys. II 4, 2215 (1994).

[23] O. Stenull, T. C. Lubensky, J. M. Adams, and M. Warner, Phys. Rev. E 78, 021705 (2008).

[24] D. Kramer and H. Finkelmann, Phys. Rev. E 78, 021704 (2008).

[25] R. Stannarius, V. Aksenov, J. Bläsing, A. Krost, M. Rössler, and R. Zentel, Phys. Chem. Chem. Phys. 8, 2293 (2006).

[26] R. Köhler, R. Stannarius, C. Tolksdorf, and R. Zentel, Appl. Phys. A: Mater. Sci. Process. 80, 381 (2005).

[27] C. M. Spillmann, B. R. Ratna, and J. Naciri, Appl. Phys. Lett. 90, 021911 (2007).

[28] H. Finkelmann, I. Kundler, E. M. Terentjev, and M. Warner, J. Phys. II 7, 1059 (1997).

[29] E. Zubarev, S. Kuptsov, T. Yuranova, R. Talroze, and H. Finkelmann, Liq. Cryst. 26, 1531 (1999).

[30] S. Conti, A. DeSimone, and G. Dolzmann, Phys. Rev. E 66, 061710 (2002). 